\title{
Effects of ascorbic acid against salt stress on the morphological and physiological parameters of Solanum melongena (L.)
}

\author{
Muhammad Irfan ${ }^{1,2 *}$, Nabeela ${ }^{1}$, Muhammad Ilyas $^{2}$ and Khaliq Ur
} Rahman $^{3}$

1. Department of Botany, Abdul Wali Khan University, Mardan-Pakistan

2. Department of Botany, University of Swabi- Swabi-Pakistan

3. Department of Chemistry, University of Swabi-Swabi-Pakistan

*Corresponding author's email: Mirfan310@yahoo.com

Citation

Muhammad Irfan, Nabeela, Muhammad Ilyas and Khaliq Ur Rahman. Effects of ascorbic acid against salt stress on the morphological and physiological parameters of Solanum melongena (L.)

. Pure and Applied Biology. Vol. 8, Issue 2, pp1425-1443. http://dx.doi.org/10.19045/bspab.2019.80083

\begin{tabular}{llll}
\hline \hline Received: 07/01/2019 & Revised: 30/04/2019 & Accepted: 02/05/2019 & Online First: 09/05/2019 \\
\hline \hline
\end{tabular}

\section{Abstract}

The recent research was done to find out the role of ascorbic acid on morphological and physiological parameters of Solanum melongena during salt stress. The experiment was carried out in pots in which the effect of salt and ascorbic acid was measured on a range of morphological and physiological parameters. In this study, ascorbic acid was applied in two ways viz. as a foliar spray on leaves or through roots in the soil at two levels $100 \mathrm{ppm}$ and 200 ppm to the plants grown under control, $60 \mathrm{mM}$ and $100 \mathrm{mM} \mathrm{NaCl}$. After sixty days of treatments it was determined that fresh and dry biomass was decreased considerably under salinity stress. Overall, it was observed that salt stress significantly down effect the morphological and physiological parameters. Decrease in the vegetative and reproductive parameters was improved with the application of ascorbic acid. The best result was obtained with the $200 \mathrm{ppm}$ ascorbic acid which significantly improved the morphological and physiological parameters of plants in both control and salt stress treatments suggesting that ascorbic acid mitigate the negative effects of salt stress.

Keywords: Ascorbic acid; Foliar spray; Morphological; Physiological; Salt stress

Introduction

Salinity refers to the presence of different kinds of salts in soil which inhibits the normal growth of plants. Salinity becomes a concern when more than normal amount of soluble salts occurs in the soil [1]. Worldwide, more than sixty million hectares of irrigated lands have been affected by salinity [2]. About $50 \%$ of the irrigated area of the world is affected by salinity and their problems are more severe in arid areas [3]. Salt stress is one of the major constraints responsible for less agricultural yield in Pakistan and all over the world. A loss of about twenty million rupees per year decrease has been reported in agriculture sector due to salinity.

Solanum melongena is the member of family Solanaceae and is an annual herb. The fruits are fleshy and have a meaty texture having less than $3 \mathrm{~cm}$ diameter. The fruits are technically called as berry which having numerous smaller seeds and are edible having bitter in taste and are 35 to 
$156 \mathrm{~cm}$ in height with large coarsely lobed leaves that are eight to $18 \mathrm{~cm}$ long and 4 to $12 \mathrm{~cm}$ broad. Their flower is whitish to purple having five-lobed corolla and yellowish stamens [4].

Ascorbic acid is growth regulator known to provide protection against a number of abiotic stresses [5] because it can influence a range of processes in plants, including seed germination [6] stomatal closure [7] ion uptake and transport [8] membrane permeability [9] photosynthetic and growth rate [10]. It also accumulates in plants under saline conditions and are known to have a vital role in salt tolerance. For example, salt-tolerant tomato cultivars had higher levels of ascorbic acid than saltsensitive cultivars [11]. Similarly, pretreatment with ascorbic acid counteracted the inhibitory effects of salt stress on growth and photosynthesis of Pisum sativum [12]. Ascorbic acid showed improvement in growth, cell division and cell enlargement of various crops [13]. However ascorbic acid is also known to induce salt tolerance through signal transduction pathways, but the factors involved in the ascorbic acid signaltransduction pathways remain to be elucidated yet [14]. Thus ascorbic acid potentially involved in counteracting salt stress. The current study was designed to analyze the effect of ascorbic acid in mitigating the effect of salinity in solanum melongena.

\section{Materials and methods}

\section{Soil preparation \& salinity treatments}

Seeds of solanum melongena were grown in pots containing 4 kilogram of soil in each. Sixty pots divided into 5 sets were used in the experiment. Each set consisted of three treatments of $\mathrm{NaCl}$ (1) control (without salt) (2) $60 \mathrm{mM}$ (3) $100 \mathrm{mM}$. Each treatment was replicated 4 times forming a total of 12 pots for overall set.

\section{Ascorbic acid treatment}

In the first set of pots no Ascorbic acid was applied while in the $2^{\text {nd }}$ and $3^{\text {rd }}$ sets of pots $100 \mathrm{ppm}$ and $200 \mathrm{ppm}$ ascorbic acid was applied respectively by mixing it in soil.
However, in the $4^{\text {th }}$ and $5^{\text {th }}$ sets of pots again 100 and 200 ppm ascorbic acid was applied using foliar spray methods. Doses of the ascorbic acid were refreshed in each month for all sets. Electrical conductivity for each treatment in each set was calculated.

\section{Seedling's analysis}

Three seedlings were grown in each pot which was irrigated with tape water. After establishment of seedlings, all pots were irrigated with $1.5 \mathrm{~L}$ of tape water after every three days. Different morphological and physiological parameters were noted at the time of harvest. Electrolytic Leakage was determined as described by [15] with few modifications.

$\operatorname{EL}(\%)=\mathrm{L}_{1} / \mathrm{L}_{2} * 100$

Relative water content was measured using the method described by [16].

$\mathrm{RWC}=(\mathrm{FW}-\mathrm{DW}) /(\mathrm{TW}-\mathrm{DW}) * 100$

Leaf water loss was determined as described by [17].

$\mathrm{LWL}(\%)=\mathrm{W}_{1}-\mathrm{W}_{2} / \mathrm{W}_{1}$

Root weight ratio, shoot weight ratio, leaf weight ratio, shoot root ratio, Leaf area ratio and Specific leaf ratio was determined as described by [18].

\section{Results and discussion \\ Plant height}

Plants of the set-I showed non-significant decrease in $100 \mathrm{mM}$ salt stress as compared to control. Similarly, set-II and -III, showed non-significant increase at $60 \mathrm{mM}$ salt stress as compared to control. Set-IV resulted non-significant decrease in saline media as compared to control however it was more prominent in $60 \mathrm{mM}$ salt stress. Set-V, $60 \mathrm{mM}$ salt stress resulted reduction as compared to control. Juxtaposition of set-II and set-III, set-II resulted slight increase in control while a prominent increase in $60 \mathrm{mM}$ salt stress as juxtaposition with setIII. Juxtaposition of set-I, set-II resulted increase and set-III resulted decrease in control and $60 \mathrm{mM}$ salt stress as juxtaposition with set-I. Juxtaposition between set-IV and set-V resulted that setIV resulted slight increase in control while a prominent increase at $60 \mathrm{mM}$ salt stress as juxtaposition with set-V. When both sets 
were juxtaposition with set-I, set-IV resulted increase and set- $\mathrm{V}$ results decrease in control and $60 \mathrm{mM}$ salt stress as juxtaposition with set-I (Table 1; Figure 1) which agrees with the earlier work of [19].

Table 1. Anova for different growth parameters in Solanum melongena grown in different $\mathrm{NaCl}$ levels and ascorbic acid doses

\begin{tabular}{|c|c|c|c|c|c|c|}
\hline Source & $\begin{array}{c}\text { Plant } \\
\text { height }\end{array}$ & $\begin{array}{c}\text { Root } \\
\text { length }\end{array}$ & $\begin{array}{c}\text { Number of } \\
\text { leaves }\end{array}$ & Leaf area & $\begin{array}{c}\text { Fresh } \\
\text { Biomass }\end{array}$ & $\begin{array}{c}\text { Dry } \\
\text { Biomass }\end{array}$ \\
\hline Application & Ns & Ns & Ns & Ns & Ns & Ns \\
\hline Ascorbic acid & Ns & Ns & Ns & Ns & Ns & Ns \\
\hline Salinity & Ns & $\mathrm{P}<0.001$ & $\mathrm{P}<0.05$ & $\mathrm{P}<0.01$ & Ns & P<0.05 \\
\hline $\begin{array}{c}\text { Application } \mathrm{x} \\
\text { Ascorbic acid }\end{array}$ & $\mathrm{Ns}$ & $\mathrm{Ns}$ & $\mathrm{Ns}$ & $\mathrm{Ns}$ & $\mathrm{Ns}$ & $\mathrm{Ns}$ \\
\hline $\begin{array}{c}\text { Application } \mathrm{x} \\
\text { salinity }\end{array}$ & $\mathrm{Ns}$ & $\mathrm{P}<0.01$ & $\mathrm{Ns}$ & $\mathrm{Ns}$ & $\mathrm{Ns}$ & $\mathrm{Ns}$ \\
\hline $\begin{array}{c}\text { Ascorbic acid } \\
\text { x salinity }\end{array}$ & $\mathrm{Ns}$ & $\mathrm{Ns}$ & $\mathrm{P}<0.05$ & $\mathrm{Ns}$ & $\mathrm{Ns}$ & $\mathrm{Ns}$ \\
\hline $\begin{array}{c}\text { Application } \mathrm{x} \\
\text { Ascorbic acid } \\
\text { x salinity }\end{array}$ & $\mathrm{Ns}$ & $\mathrm{Ns}$ & $\mathrm{P}<0.05$ & $\mathrm{Ns}$ & $\mathrm{Ns}$ & $\mathrm{Ns}$ \\
\hline
\end{tabular}

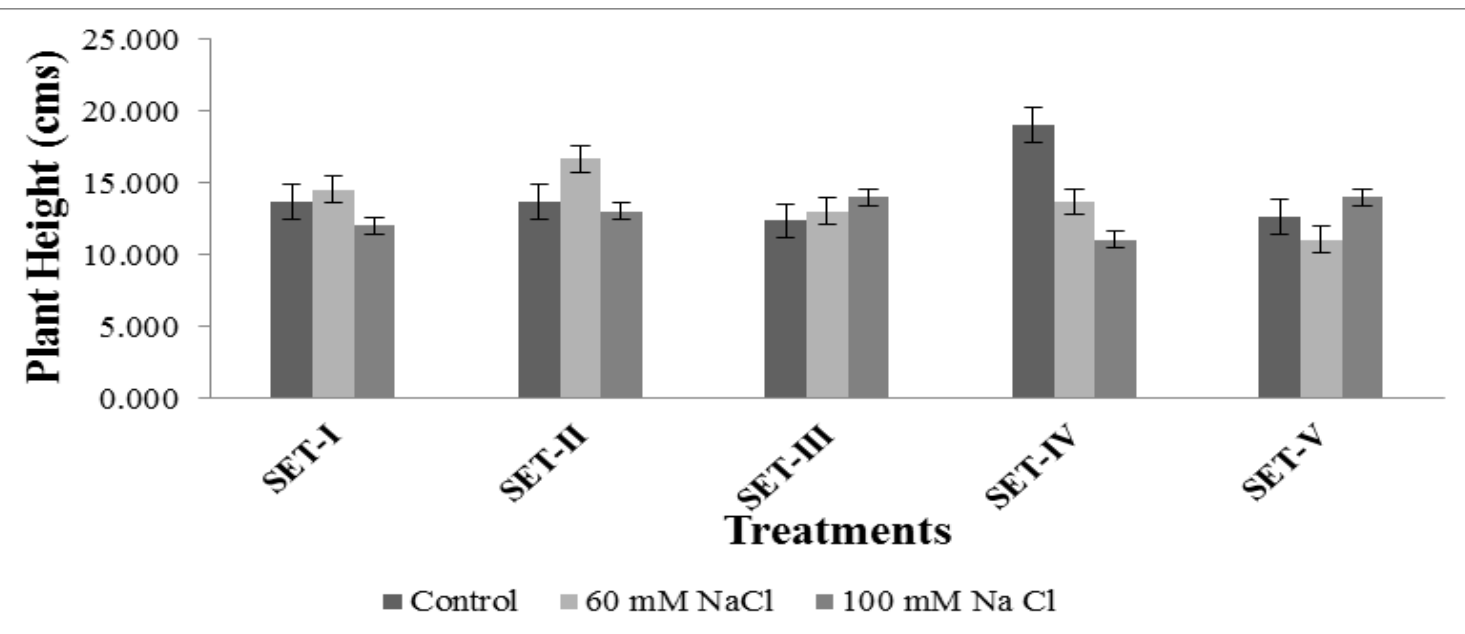

Figure 1. Effects of Ascorbic acid and different $\mathrm{NaCl}$ concentrations on plant height (cms) of Solanum melongena. Set-I: Without ascorbic acid, Set-II: $0.25 \mathrm{ppm}$ ascorbic acid applied through roots in soil, Set-III: $0.50 \mathrm{ppm}$ ascorbic acid applied through roots in soil, Set-IV: $0.25 \mathrm{ppm}$ ascorbic acid applied as a foliar spray, Set-V: $0.50 \mathrm{ppm}$ ascorbic acid applied as a foliar spray

\section{Root length}

Plants in set-I resulted significant $(\mathrm{P}<0.001)$ increase in saline media viz.60mM salt stress as juxtaposition with control. In set-II salt stress resulted significant $\quad(\mathrm{P}<0.001) \quad$ increase as juxtaposition with control. Plants in set-III resulted significant $(\mathrm{P}<0.001)$ decrease in $60 \mathrm{mM}$ salt stress as juxtaposition with control. Set-IV and set-V resulted significant $(\mathrm{P}<0.001)$ decrease in both salt stress as juxtaposition with control. Juxtaposition of set-II and set-III, set-II resulted decrease in control and $60 \mathrm{mM}$ salt stress as juxtaposition with set-III. Juxtaposition of set-II and set-III with set-I both sets resulted increase in $100 \mathrm{mM}$ salt stress as juxtaposition with set-I. Juxtaposition of set-IV and set-V resulted that Set-IV resulted increase in all 
treatments as juxtaposition with set-V. Juxtaposition of set-IV and set-V with setI, Set-IV resulted increase in control and set-V resulted significant decrease in saline media as juxtaposition with set-I (Table 1; Figure 2) which agrees with the earlier work of [20].

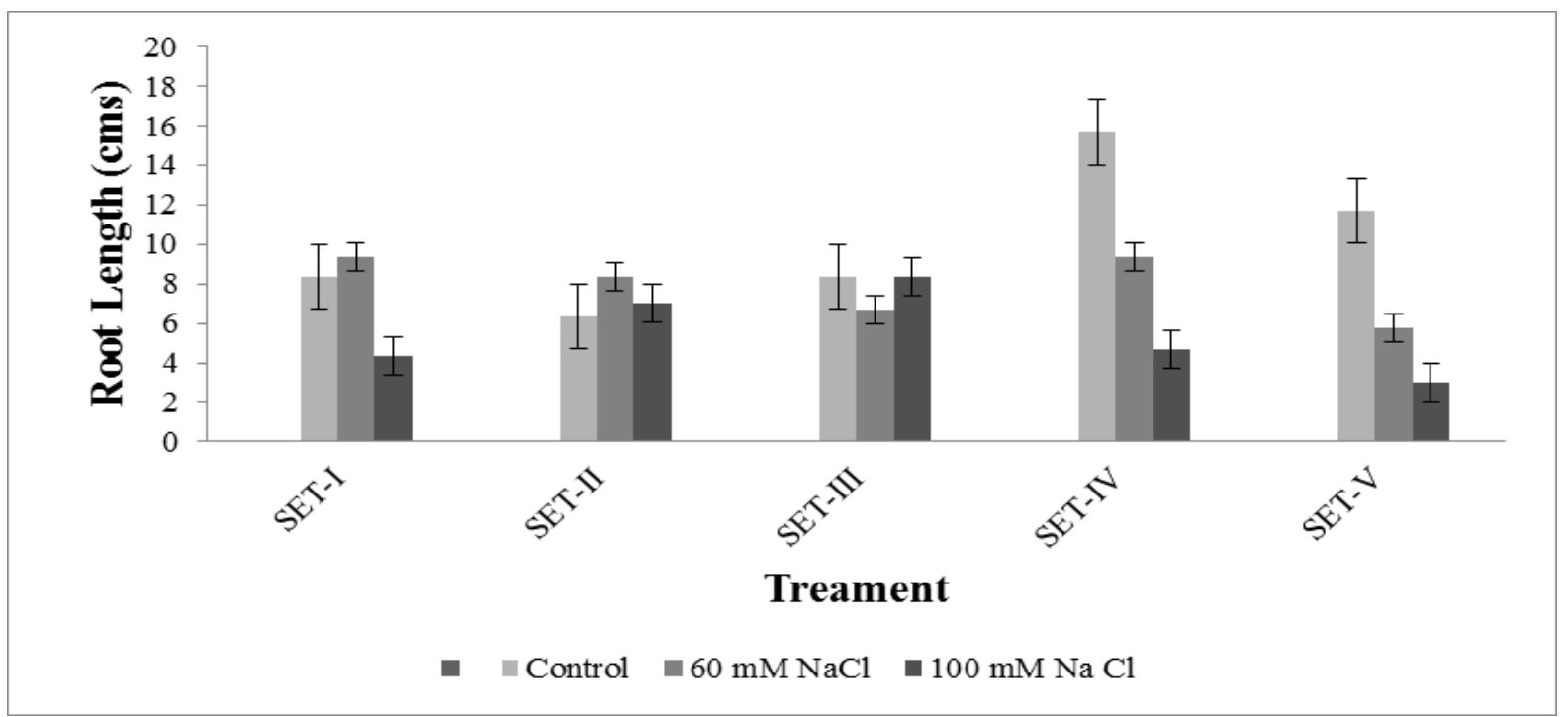

Figure 2. Effects of Ascorbic acid and different $\mathrm{NaCl}$ concentrations on root length $(\mathrm{cms})$ of Solanum melongena. Set-I: Without ascorbic acid, Set-II: $0.25 \mathrm{ppm}$ ascorbic acid applied through roots in soil, Set-II: $0.50 \mathrm{ppm}$ ascorbic acid applied through roots in soil, Set-IV; 0.25 ppm ascorbic acid applied as a foliar spray, Set-V: $0.50 \mathrm{ppm}$ ascorbic acid applied as a foliar spray

\section{Number of leaves}

Plants of set-I resulted significant $(\mathrm{P}<0.05)$ decrease in $60 \mathrm{mM}$ and $100 \mathrm{mM}$ salt stress as juxtaposition with control. Set-II grown under $60 \mathrm{mM}$ salt stress resulted significant $(\mathrm{P}<0.05)$ increase as juxtaposition with control. Set - III resulted significant $(\mathrm{P}<0.05)$ increase in $100 \mathrm{mM}$ salt stress treatment as juxtaposition with control. SetIV resulted significant $(\mathrm{P}<0.05)$ reduction in saline media as juxtaposition with control. Set-V, 60mM salt stress resulted increase while $100 \mathrm{mM}$ salt stress exhibited decrease as juxtaposition with control. This result was statistically significant $(\mathrm{P}<0.05)$. Juxtaposition of set-II and set-III, set-II resulted increase in control and $60 \mathrm{mM}$ salt stress as juxtaposition with set-III. Juxtaposition of set-II and set-III with set-I, Set-II resulted increase in $60 \mathrm{mM}$ salt stress and set-III resulted increase in both salinity levels $60 \mathrm{mM}$ and $100 \mathrm{mM}$ salt stress treatments as juxtaposition with set-I. Juxtaposition of set-IV and set-V results that Set-IV resulted increase in control and in high salinity treatment viz. $100 \mathrm{mM}$ salt stresses as juxtaposition with set-V. Juxtaposition of set-IV and set-V with setI, Set-IV resulted increase in control and $60 \mathrm{mM}$ salt stress and set-V resulted increase only at $60 \mathrm{mM}$ salt stress as Juxtaposition with set-I (Table 1; Figure 3). 


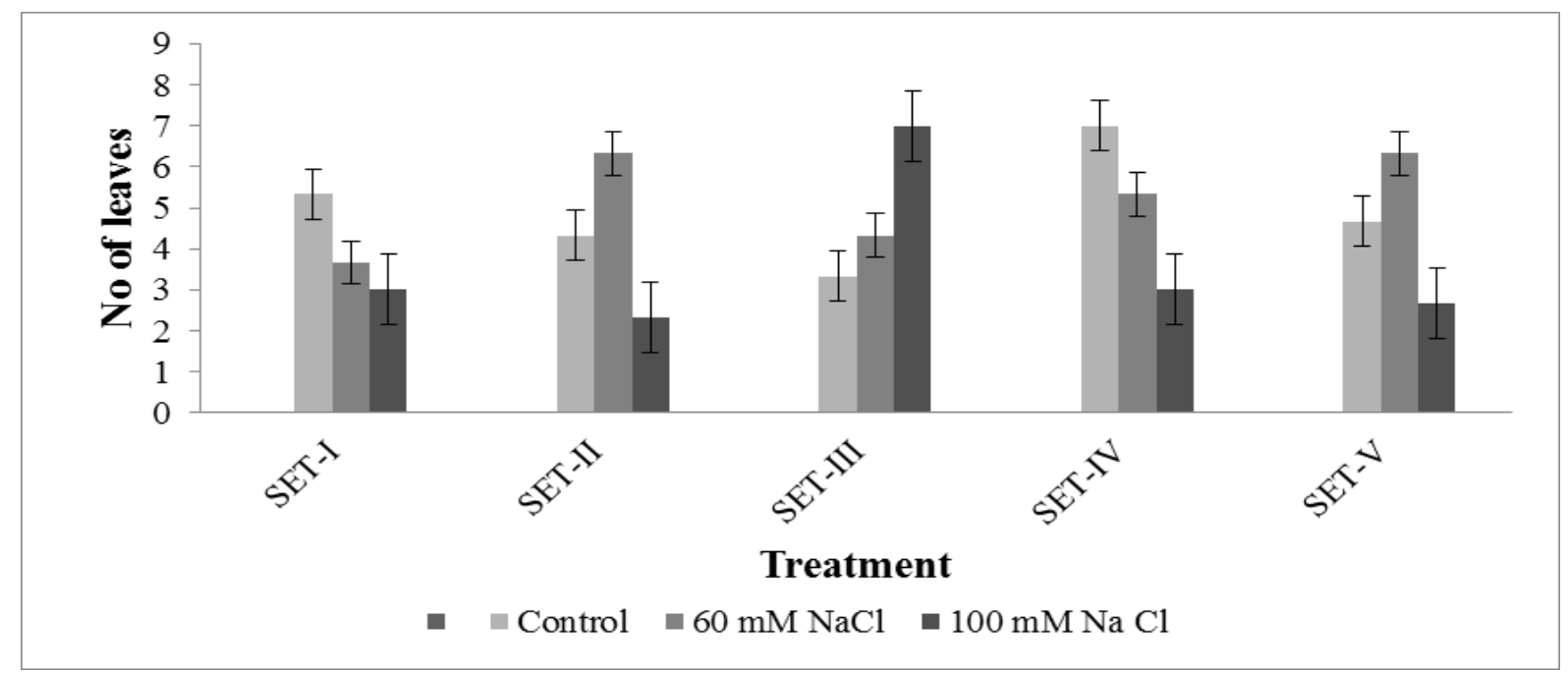

Figure 3. Effects of Ascorbic acid and different $\mathrm{NaCl}$ concentrations on number of leaves (cms) of Solanum melongena. Set-I: Without ascorbic acid, Set-II: $0.25 \mathrm{ppm}$ ascorbic acid applied through roots in soil, Set-II: $0.50 \mathrm{ppm}$ ascorbic acid applied through roots in soil, Set-IV: $0.25 \mathrm{ppm}$ ascorbic acid applied as a foliar spray, Set-V: $0.50 \mathrm{ppm}$ ascorbic acid applied as a foliar spray

\section{Leaf area}

Plants grown in set-I and set-II resulted a prominent significant $(\mathrm{P}<0.01)$ increase in $60 \mathrm{mM}$ salt stress and a prominent significant $(\mathrm{P}<0.01)$ decrease in $100 \mathrm{mM}$ salt stress as juxtaposition with control. SetIII grown under $60 \mathrm{mM}$ salt stress resulted significant $\quad(\mathrm{P}<0.05) \quad$ increase as juxtaposition with control. Set-IV resulted significant $(\mathrm{P}<0.05)$ reduction in saline media as juxtaposition with control. Set-V, $60 \mathrm{mM}$ salt stress resulted non-significant increase as juxtaposition with control. Juxtaposition of set-II and set-III, set-II resulted increase only in control and $60 \mathrm{mM}$ salt stress as juxtaposition with set-III. Juxtaposition of set-II and set-III with set-I, Set-II resulted increase in all treatments and set-III in $100 \mathrm{mM}$ salt stress as juxtaposition with set-I. Juxtaposition between set-IV and set-V resulted that Set-IV resulted increase in control as juxtaposition with setV. Juxtaposition of set-IV and set-V with set-I, Set-IV resulted increase in control and $100 \mathrm{mM}$ salt stress and set-V in $100 \mathrm{mM}$ salt stress as juxtaposition with set-I (Table 1;
Figure 4) which agrees with the earlier work of [21].

\section{Fresh and dry biomass}

Plants of set-I resulted significant $(\mathrm{P}<0.05)$ decrease in saline media as juxtaposition with control. Set-II and III resulted significant $(\mathrm{P}<0.05)$ increase in $60 \mathrm{mM}$ salt stress as juxtaposition with control. Set-IV and $\mathrm{V}$ resulted significant $(\mathrm{P}<0.05)$ decrease in $60 \mathrm{mM}$ salt stress as juxtaposition with control. Juxtaposition of set-II and set-III, set-III resulted slight increase in all treatments as juxtaposition with set-II. Juxtaposition of set-II and setIII with set-I, Set-II resulted decrease in all treatments and set-III in control and $60 \mathrm{mM}$ salt stress as juxtaposition with set-I. Juxtaposition of set-IV and set-V resulted that Set-IV showed increase in control and $60 \mathrm{mM}$ salt stress as juxtaposition with setV. Juxtaposition of set-IV and set-V with set-I, Set-IV resulted increase in control and set- $\mathrm{V}$ in control and $100 \mathrm{mM}$ salt stress as juxtaposition with set-I (Table 1; Figure 5 \& 6). 


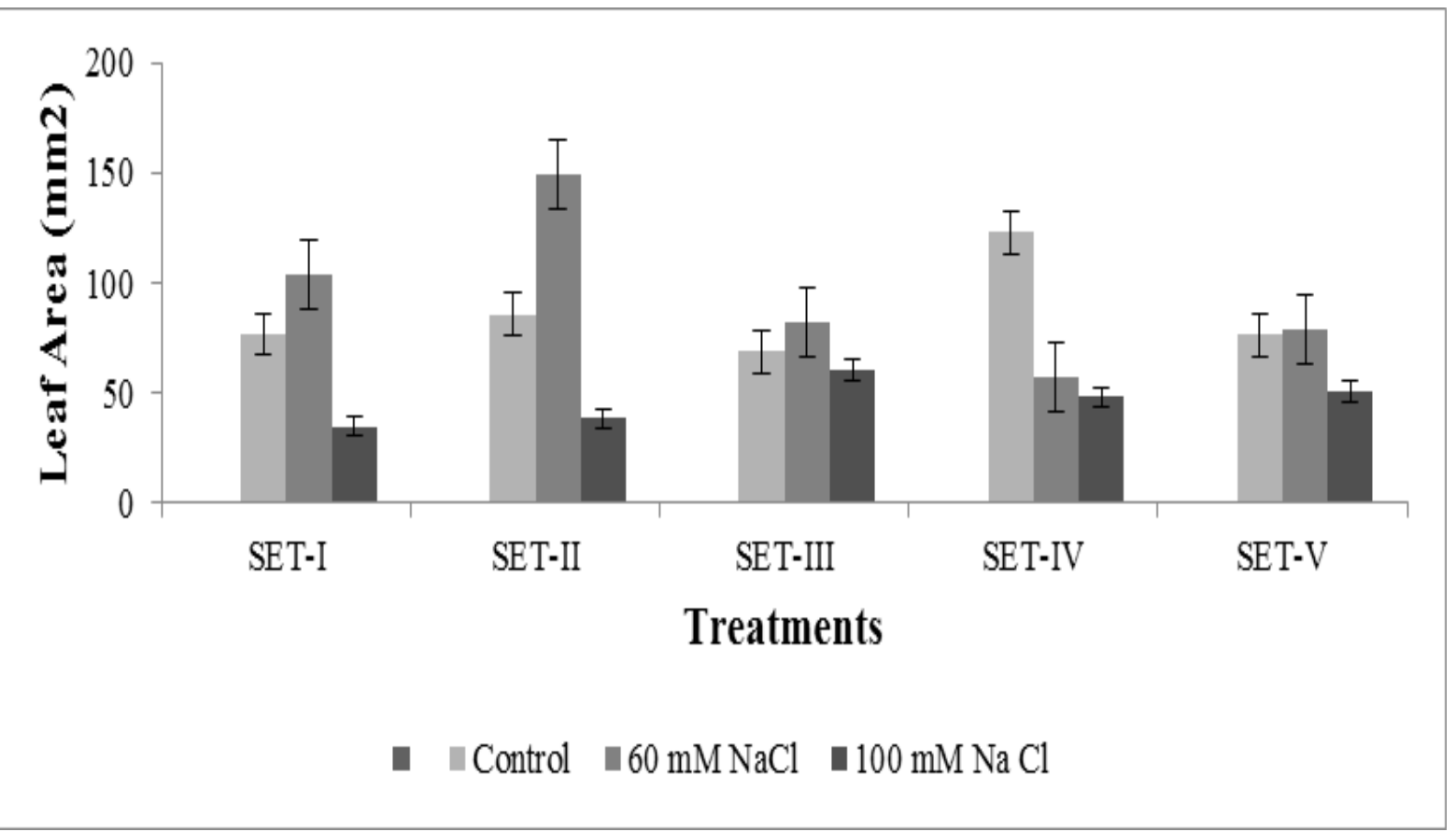

Figure 4. Effects of Ascorbic acid and different $\mathrm{NaCl}$ concentrations on leaf area $(\mathrm{cms})$ of Solanum melongena. Set-I: Without ascorbic acid, set-II: $0.25 \mathrm{ppm}$ ascorbic acid applied through roots in soil, Set-II: $\mathbf{0 . 5 0} \mathrm{ppm}$ ascorbic acid applied through roots in soil, Set-IV: $0.25 \mathrm{ppm}$ ascorbic acid applied as a foliar spray, Set-V: $0.50 \mathrm{ppm}$ ascorbic acid applied as a foliar spray

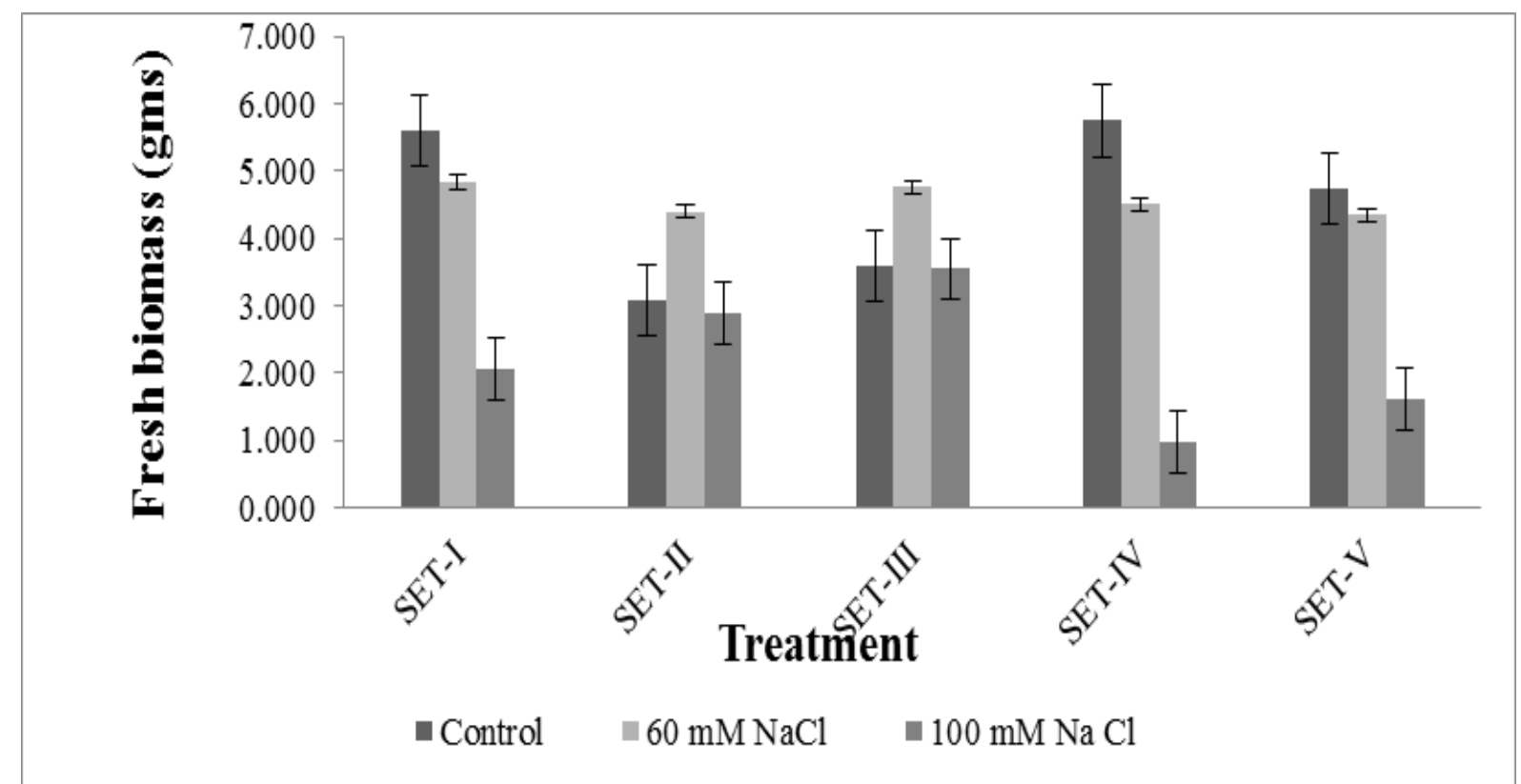

Figure 5. Effects of Ascorbic acid and different $\mathrm{NaCl}$ concentrations on plant fresh biomass (cms) of Solanum melongena. Set-I: Without ascorbic acid, Set-II: 0.25 ppm ascorbic acid applied through roots in soil, Set-II: $0.50 \mathrm{ppm}$ ascorbic acid applied through roots in soil, Set-IV: $0.25 \mathrm{ppm}$ ascorbic acid applied as a foliar spray, Set-V: $0.50 \mathrm{ppm}$ ascorbic acid applied as a foliar spray 


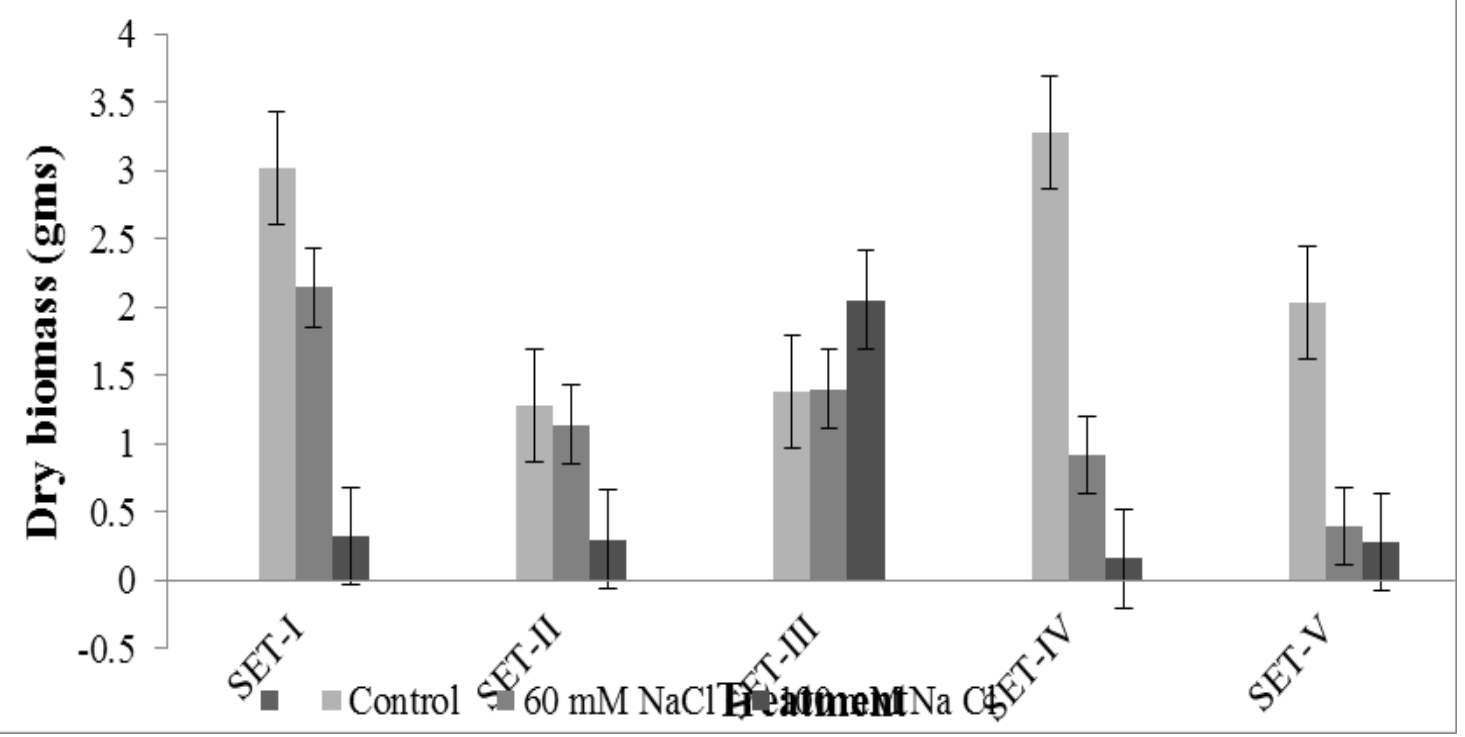

Figure 6. Effects of Ascorbic acid and different $\mathrm{NaCl}$ concentrations on plantdry biomass (cms) of Solanum melongena. Set-I: Without ascorbic acid, Set-II: 0.25 ppm ascorbic acid applied through roots in soil, Set-III: $0.50 \mathrm{ppm}$ ascorbic acid applied through roots in soil, Set-IV: $0.25 \mathrm{ppm}$ ascorbic acid applied as a foliar spray, Set-V: $0.50 \mathrm{ppm}$ ascorbic acid applied as a foliar spray

\section{Electrolyte leakage}

In set-I 100mM salt stress resulted nonsignificant increase as juxtaposition with control. Set-II resulted non- significant increase in saline media as juxtaposition with control. Set-III viz. $60 \mathrm{mM}$ salt stress resulted non-significant increase as juxtaposition with control. Set-IV and set$\mathrm{V}$ resulted significant $(\mathrm{P}<0.05)$ decrease in saline media as juxtaposition with control. Juxtaposition of set-II and set-III, set-III resulted increase in control and $60 \mathrm{mM}$ salt stress as juxtaposition with set-II. Juxtaposition of set-II and set-III with set-I both sets resulted increase at $60 \mathrm{mM}$ salt stress as juxtaposition with set-I. Juxtaposition between set-IV and set-V resulted that Set-V resulted increase in all treatments as juxtaposition with set-IV. Juxtaposition of set-IV and set-V with setI, Set-IV resulted decrease in control and $100 \mathrm{mM}$ salt stress and set-V resulted increase in $100 \mathrm{mM}$ salt stress as juxtaposition with set-II (Table 2).

\section{Leaf water loss}

Plants grown in set-I results a prominent increase in saline media as juxtaposition with control. Set-II resulted significant $(\mathrm{P}<0.05)$ increase at $100 \mathrm{mM}$ salt stress as juxtaposition with control. Set-III resulted significant $(\mathrm{P}<0.05)$ decrease in $60 \mathrm{mM}$ salt stress as juxtaposition with control. Set-IV resulted increase in saline media as juxtaposition with control. Set-V resulted significant $(\mathrm{P}<0.05)$ decrease in saline media as juxtaposition with control. Juxtaposition of set-II and set-III, set-II resulted increase in all treatments as juxtaposition with set-III. Juxtaposition of set-II and set-III with set-I, Set- II resulted increase in all treatments and set-III resulted increase in control and $60 \mathrm{mM}$ salt stress as juxtaposition with set-I. Juxtaposition between set-IV and set-V resulted that Set-IV resulted increase in saline media as juxtaposition with set-V. Juxtaposition of set-IV and set-V with setI, Set-IV resulted increase in control and $100 \mathrm{mM}$ salt stress and set-V resulted decrease in saline media as juxtaposition with set-I (Table 2). 


\section{Relative water content}

Plants of set-I exhibited non-significant increase in $100 \mathrm{mM}$ salt stress as juxtaposition with control. Set-II resulted non-significant increase at $60 \mathrm{mM}$ salt stress as juxtaposition with control. Set-III resulted non-significant decrease at $100 \mathrm{mM}$ salt stress as juxtaposition with control. SetIV resulted non-significant increase in $60 \mathrm{mM}$ salt stress as juxtaposition with control. Set-V resulted non-significant increase in $60 \mathrm{mM}$ salt stress as juxtaposition with control. Juxtaposition of set-II and set-III, set-II resulted prominent increase in saline media as juxtaposition with set-III. Juxtaposition of set-II and setIII with set-I, Set- II resulted prominent increased at $60 \mathrm{mM}$ salt stress and set-III resulted increase in control and $60 \mathrm{mM}$ salt stress as juxtaposition with set-I. Juxtaposition between set-IV and set-V resulted that Set-IV resulted increase in $60 \mathrm{mM}$ salt stress as juxtaposition with setV. Juxtaposition of set-IV and set-V with set-I, Set-IV resulted increase in control and $60 \mathrm{mM}$ salt stress and set-V resulted increase in $60 \mathrm{mM}$ salt stress as juxtaposition with set-I (Table 2).

Table 2. Anova for effects of ascorbic acid and different $\mathrm{NaCl}$ concentrations on relative water content, Electrolyte leakage and leaf water loss of Solanum melongena

Set-I= Without Ascorbic acid

\begin{tabular}{|c|c|c|c|}
\hline Treatment & Relative water content & Electrolyte leakage & Leaf water loss \\
\hline Control, Mean, SE & $52.226 \mathrm{a} \pm 23.726$ & $27.062 \mathrm{a} \pm 14.918$ & $29.414 \mathrm{a} \pm 1.711$ \\
\hline $\begin{array}{c}60 \mathrm{mM} \mathrm{NaCl}, \\
\text { Mean, SE }\end{array}$ & $51.376 \mathrm{a} \pm 13.502$ & $46.076 \mathrm{a} \pm 1.458$ & $23.156 \mathrm{a} \pm 2.923$ \\
\hline$\%(+/-)$ & $(-1.627)$ & $(+70.262)$ & $(-21.274)$ \\
\hline $\begin{array}{c}100 \mathrm{mM} \mathrm{NaCl}, \\
\mathrm{Mean}, \mathrm{SE}\end{array}$ & $79.483 \mathrm{a} \pm 18.348$ & $35.707 \mathrm{a} \pm 6.83$ & $33.237 \mathrm{a} \pm 16.618$ \\
\hline$\%(+/-)$ & $(+52.189)$ & $(+31.945)$ & $(+12.997)$ \\
\hline $\mathrm{LSD}_{0.05}$ & 65.716 & 32.91 & 3.884 \\
\hline
\end{tabular}

Table 2....... (Contd)

Set- II $=0.25 \mathrm{ppm}$ Ascorbic acid applied through roots in soil

\begin{tabular}{|c|c|c|c|}
\hline Treatment & Relative Water Content & Electrolyte leakage & Leaf Water Loss \\
\hline $\begin{array}{c}\text { Control, Mean, SE } \\
60 \mathrm{mM} \mathrm{NaCl}, \text { Mean, } \\
\mathrm{SE}\end{array}$ & $41.006 \mathrm{a} \pm 4.620$ & $59.183 \mathrm{a} \pm 17.711$ & $29.212 \mathrm{a} \pm 1.888$ \\
\hline$\%(+/-)$ & $52.333 \mathrm{a} \pm 6.633$ & $47.243 \mathrm{a} \pm 1.102$ & $1377.439 \mathrm{~b} \pm 140.612$ \\
\hline $\begin{array}{c}100 \mathrm{mM} \mathrm{NaCl}, \text { Mean, } \\
\mathrm{SE}\end{array}$ & $(+27.621)$ & $(-20.174)$ & $(+4615.18)$ \\
\hline$\%(+/-)$ & $56.29 \mathrm{a} \pm 9.336$ & $101.873 \mathrm{a} \pm 46.849$ & $27.042 \mathrm{~b} \pm 3.124$ \\
\hline $\mathrm{LSD}_{0.05}$ & $(+37.27)$ & $(+72.131)$ & $(-7.429)$ \\
\hline
\end{tabular}

Table 2........ ( Contd)

Set-III $0.50 \mathrm{ppm}$ Ascorbic acid applied through roots in soil

\begin{tabular}{|c|c|c|c|}
\hline Treatment & Relative water content & Electrolyte leakage & Leaf water loss \\
\hline Control, Mean, SE & $51.14 \mathrm{a} \pm 11.14$ & $41.775 \mathrm{a} \pm 1.065$ & $43.839 \mathrm{a} \pm 6.756$ \\
\hline $\begin{array}{c}60 \mathrm{mM} ~ \mathrm{NaCl}, \mathrm{Mean}, \\
\mathrm{SE}\end{array}$ & $68.16 \mathrm{a} \pm 13.517$ & $22.53 \mathrm{a} \pm 1.506$ & $33.191 \mathrm{ab} \pm 0.275$ \\
\hline$\%(+/-)$ & $(+33.281)$ & $(+46.069)$ & $(-24.287)$ \\
\hline $\begin{array}{c}100 \mathrm{mM} ~ \mathrm{NaCl}, \mathrm{Mean}, \\
\mathrm{SE}\end{array}$ & $53.55 \mathrm{a} \pm 17.372$ & $44.323 \mathrm{~b} \pm 5.958$ & $1.628 \mathrm{~b} \pm 1.631$ \\
\hline$\%(+/-)$ & $(+4.712)$ & $(-6.098)$ & $(-38.178)$ \\
\hline $\mathrm{LSD}_{0.05}$ & 49.29 & 12.462 & 13.897 \\
\hline
\end{tabular}


Table 2........ ( Contd)

Set-IV 0.25 ppm Ascorbic acid applied as a foliar spray

\begin{tabular}{|c|c|c|c|}
\hline Treatment & Relative Water Content & Electrolyte leakage & Leaf Water Loss \\
\hline Control, Mean, SE & $42.583 \mathrm{a} \pm 10.993$ & $39.412 \mathrm{a} \pm 11.031$ & $30.945 \mathrm{a} \pm 1.937$ \\
\hline $\begin{array}{c}\text { 60mM NaCl, } \\
\text { Mean, SE }\end{array}$ & $61.086 \mathrm{a} \pm 16.775$ & $44.653 \mathrm{a} \pm 5.184$ & $36.162 \mathrm{a} \pm 7.481$ \\
\hline$\%(+/-)$ & $(+43.452)$ & $(+13.296)$ & $(+16.857)$ \\
\hline $\begin{array}{c}100 \mathrm{mM} \mathrm{NaCl}, \\
\text { Mean, SE }\end{array}$ & $74.726 \mathrm{a} \pm 12.229$ & $45.026 \mathrm{a} \pm 0.973$ & $27.979 \mathrm{a} \pm 1.631$ \\
\hline$\%(+/-)$ & $(+75.483)$ & $(+14.244)$ & $(-9.583)$ \\
\hline $\mathrm{LSD}_{0.05}$ & 46.933 & 24.429 & 15.780 \\
\hline
\end{tabular}

Table 2........ ( Contd)

Set-V $0.50 \mathrm{ppm}$ Ascorbic acid applied as a foliar spray

\begin{tabular}{|c|c|c|c|}
\hline Treatment & Relative water content & Electrolyte leakage & Leaf water loss \\
\hline Control, Mean, SE & $65.146 \mathrm{a} \pm 4.264$ & $41.107 \mathrm{a} \pm 4.807$ & $26.522 \mathrm{a} \pm 0.927$ \\
\hline $\begin{array}{c}60 \mathrm{mM} \mathrm{NaCl}, \\
\mathrm{Mean}, \mathrm{SE}\end{array}$ & $78.723 \mathrm{a} \pm 17.773$ & $26.863 \mathrm{~b} \pm 2.957$ & $313.118 \mathrm{~b} \pm 14.985$ \\
\hline$\%(+/-)$ & $(+20.84)$ & $(-34.651)$ & $(+1080.593)$ \\
\hline $\begin{array}{c}100 \mathrm{mM} \mathrm{NaCl}, \\
\mathrm{Mean}, \mathrm{SE}\end{array}$ & $78.576 \mathrm{a} \pm 17.091$ & $29.126 \mathrm{~b} \pm 1.803$ & $99.251 \mathrm{~b} \pm 70.537$ \\
\hline$\%(+/-)$ & $(+20.615)$ & $(-29.145)$ & $(+274.219)$ \\
\hline $\mathrm{LSD}$ & 49.996 & 11.839 & 144.083 \\
\hline
\end{tabular}

Means followed by different letters in the same column differ significantly at $95 \%$ probability level according to New Duncan's Multiple Range Test. Figures in parentheses indicate \% promotion (+) and reduction (-) over control

\section{Shoot root ratio}

Plants of set-I results decrease in $60 \mathrm{mM}$ salt stress as juxtaposition with control. Set-II and set-III resulted decrease in saline media as juxtaposition with control. Set-IV resulted increase in $60 \mathrm{mM}$ salt stress as juxtaposition with control. Set-V exhibited increase in $100 \mathrm{mM}$ salt stress as juxtaposition with control. Juxtaposition of set-II and set-III, set-II resulted reduction in control as well as in saline media as juxtaposition with set-III. Juxtaposition of
set-II and set-III with set-I, both sets resulted increase in control and $100 \mathrm{mM}$ salt stress juxtaposition with set-I. Juxtaposition between set-IV and set-V resulted that Set-IV showed increase in control and a prominent increased in $60 \mathrm{mM}$ salt stress as juxtaposition with set-V. Juxtaposition of set-IV and set-V with setI, Set-IV resulted increased in control and a prominent increase in $60 \mathrm{mM}$ salt stress and set- $V$ resulted reduction in $60 \mathrm{mM}$ salt stress juxtaposition with set-I (Table 3).

Table 3. Effect of Ascorbic acid and different $\mathrm{NaCl}$ concentrations on shoot/root ratio of Solanum melongena.

Set-I= Without Ascorbic acid

\begin{tabular}{|c|c|c|c|}
\hline Treatment & Shoot Dry Wt (gms) & Root Dry Wt (gms) & Shoot/Root Ratio \\
\hline Control, Mean, SE & $2.64 \mathrm{a} \pm .359$ & $3.021 \mathrm{a} \pm 0.42$ & $7.254 \mathrm{a} \pm 0.962$ \\
\hline $\begin{array}{c}60 \mathrm{mM} \mathrm{NaCl}, \\
\mathrm{Mean}, \mathrm{SE}\end{array}$ & $1.314 \mathrm{a} \pm 0.574$ & $2.142 \mathrm{a} \pm 0.518$ & $15.71 \mathrm{a} \pm 10.271$ \\
\hline$\%(+/-)$ & $(-50.227)$ & $(-29.096)$ & $(-116.57)$ \\
\hline $\begin{array}{c}100 \mathrm{mM} \mathrm{NaCl}, \\
\mathrm{Mean}, \mathrm{SE}\end{array}$ & $0.134 \mathrm{~b} \pm 0.11$ & $0.319 \mathrm{~b} \pm 0.173$ & $8.172 \mathrm{a} \pm 2.927$ \\
\hline$\%(+/-)$ & $(-94.924)$ & $(-89.44)$ & $(-12.655)$ \\
\hline $\mathrm{LSD}_{0.05}$ & 1.371 & 1.38 & 21.425 \\
\hline
\end{tabular}


Table 3........ ( Contd)

Set-II $=0.25 p p m$ Ascorbic acid applied through roots in soil

\begin{tabular}{|c|c|c|c|}
\hline Treatment & Shoot Dry Wt $($ gms) & Root Dry Wt (gms) & Shoot/Root Ratio \\
\hline Control, Mean, SE & $1.074 \mathrm{a} \pm 0.435$ & $1.276 \mathrm{a} \pm 0.327$ & $50.579 \mathrm{a} \pm 47.272$ \\
\hline $\begin{array}{c}\text { 60mM NaCl, } \\
\text { Mean, SE }\end{array}$ & $0.910 \mathrm{a} \pm 0.230$ & $1.137 \mathrm{a} \pm 0.231$ & $4.020 \pm 0.996$ \\
\hline$\%(+/-)$ & $(-15.27)$ & $(-10.893)$ & $(-92.052)$ \\
\hline $\begin{array}{c}100 \mathrm{mM} \mathrm{NaCl}, \\
\mathrm{Mean}, \mathrm{SE}\end{array}$ & $0.285 \mathrm{a} \pm 0.245$ & $0.298 \mathrm{a} \pm 0.245$ & $22.820 \pm 19.67$ \\
\hline$\%(+/-)$ & $(-73.463)$ & $(-76.645)$ & $(-54.882)$ \\
\hline $\mathrm{LSD}_{0.05}$ & 1.099 & 0.964 & 102.314 \\
\hline
\end{tabular}

Table 3....... ( Contd)

Set-III= 0.50ppm Ascorbic acid applied through roots in soil

\begin{tabular}{|c|c|c|c|}
\hline Treatment & Shoot Dry Wt (gms) & Root Dry Wt (gms) & Shoot/Root Ratio \\
\hline Control, Mean, SE & $1.241 \mathrm{a} \pm 0.532$ & $1.376 \mathrm{a} \pm 0.472$ & $61.103 \pm 57.407$ \\
\hline $\begin{array}{c}\text { 60mM NaCl, } \\
\text { Mean, SE }\end{array}$ & $1.142 \mathrm{a} \pm 0.071$ & $1.398 \mathrm{a} \pm 0.062$ & $6.265 \pm 2.979$ \\
\hline$\%(+/-)$ & $(-7.977)$ & $(-1.598)$ & $(-89.746)$ \\
\hline $\begin{array}{l}\text { 100mM NaCl, } \\
\text { Mean, SE }\end{array}$ & $1.891 \mathrm{a} \pm 0.523$ & $2.05 \mathrm{a} \pm 0.597$ & $29.826 \pm 19.404$ \\
\hline$\%(+/-)$ & $(-52.377)$ & $(-48.982)$ & $(-51.187)$ \\
\hline $\operatorname{LSD}_{0.05}$ & 1.499 & 1.527 & 121.214 \\
\hline
\end{tabular}

Table 3........ ( Contd)

Set-IV= 0.25ppm Ascorbic acid applied as a foliar spray

\begin{tabular}{|c|c|c|c|}
\hline Treatment & Shoot Dry Wt $($ gms) & Root Dry Wt (gms) & Shoot/Root Ratio \\
\hline Control, Mean, SE & $2.435 \mathrm{a} \pm 0.527$ & $3.278 \mathrm{a} \pm 0.759$ & $15.549 \mathrm{a} \pm 6.696$ \\
\hline $\begin{array}{c}60 \mathrm{mM} \mathrm{NaCl}, \\
\text { Mean, SE }\end{array}$ & $0.717 \mathrm{~b} \pm 0.213$ & $0.915 \mathrm{~b} \pm 0.262$ & $309.149 \mathrm{a} \pm 193.971$ \\
\hline$\%(+/)$ & $(-70.554)$ & $(-72.086)$ & $(+1888.224$ \\
\hline $\begin{array}{c}100 \mathrm{mM} \mathrm{NaCl}, \\
\text { Mean, SE }\end{array}$ & $0.12 \mathrm{~b} \pm 0.07$ & $0.158 \mathrm{~b} \pm 0.106$ & $0.661 \mathrm{a} \pm 0.381$ \\
\hline$\%(+/-)$ & $(-95.071)$ & $(-95.179)$ & $(-95.748)$ \\
\hline $\mathrm{LSD}_{0.05}$ & 1.186 & 1.619 & 387.766 \\
\hline
\end{tabular}

Table 3........ ( Contd)

Set-V=0.50ppm Ascorbic acid applied as a foliar spray

\begin{tabular}{|c|c|c|c|}
\hline Treatment & Shoot Dry Wt (gms) & Root Dry Wt (gms) & Shoot/Root Ratio \\
\hline Control, Mean, SE & $1.722 \mathrm{a} \pm 0.608$ & $2.029 \mathrm{a} \pm 0.747$ & $8.19 \mathrm{a} \pm 4.125$ \\
\hline $\begin{array}{c}\text { 60mM NaCl, } \\
\text { Mean, SE }\end{array}$ & $0.342 b \pm 0.042$ & $0.395 b \pm 0.029$ & $7.427 b \pm 2.09$ \\
\hline$\%(+/-)$ & $(-80.139)$ & $(-80.532)$ & $(-9.316)$ \\
\hline $\begin{array}{l}\text { 100mM NaCl, } \\
\text { Mean, SE }\end{array}$ & $0.265 b \pm 0.006$ & $0.276 b \pm 0.007$ & $23 \mathrm{~b} \pm 0.059$ \\
\hline$\%(+/-)$ & $(-84.61)$ & $(-86.397)$ & $(+180.83)$ \\
\hline $\mathrm{LSD}_{0.05}$ & 1.219 & 1.493 & 9.241 \\
\hline
\end{tabular}

Means followed by different letters in the same column differ significantly at $95 \%$ probability level according to New Duncan's Multiple Range Test. Figures in parentheses indicate \% promotion (+) and reduction (-) over control

\section{Root weight ratio}

Plants of set-I exhibited a slight increase in $100 \mathrm{mM}$ salt stress as juxtaposition with control. Set-II resulted reduction in salt stress concentrations as juxtaposition with control. Set-III resulted increase at $60 \mathrm{mM}$ salt stress as juxtaposition with control. Set-IV resulted increase at $100 \mathrm{mM}$ salt 
stress as juxtaposition with control. Set-V resulted decrease in saline media as juxtaposition with control. Juxtaposition of set-II and set-III, set-II resulted increase as juxtaposition with set-III. Juxtaposition of set-II and set-III with set-I, set-II resulted increase in both saline media and control and set-III exhibited reduction at $100 \mathrm{mM}$ salt stress as juxtaposition with set-I.
Juxtaposition between set-IV and set-V resulted that Set-IV resulted prominent increased in $100 \mathrm{mM}$ salt stress as juxtaposition with set-V. Juxtaposition of set-IV and set-V with set-I, Set-IV resulted a prominent increase at $100 \mathrm{mM}$ salt stress and set- $\mathrm{V}$ reduction in saline media as juxtaposition with set-I (Table 4).

Table 4. Effect of Ascorbic acid and different $\mathrm{NaCl}$ concentrations on root weight ratio of Solanum melongena.

Set-I= Without Ascorbic acid

\begin{tabular}{|c|c|c|c|}
\hline Treatment & $\begin{array}{c}\text { Total Root Dry Wt } \\
(\mathbf{g m s})\end{array}$ & Total Plant Dry Wt (gms) & Root Weight Ratio \\
\hline Control, Mean, SE & $0.381 \mathrm{a} \pm 0.075$ & $3.021 \mathrm{a} \pm 0.42$ & $0.124 \mathrm{a} \pm 0.014$ \\
\hline $\begin{array}{c}\text { 60mM NaCl, } \\
\text { Mean, SE }\end{array}$ & $0.196 \mathrm{ab} \pm 0.055$ & $2.142 \mathrm{a} \pm 0.518$ & $0.112 \mathrm{a} \pm 0.043$ \\
\hline$\%(+/-)$ & $(-48.556)$ & $(-29.096)$ & $(-9.677)$ \\
\hline $\begin{array}{c}100 \mathrm{mM} \mathrm{NaCl}, \\
\text { Mean, SE }\end{array}$ & $0.075 \mathrm{~b} \pm 0.72$ & $0.319 \mathrm{~b} \pm 0.173$ & $0.155 \mathrm{a} \pm 0.072$ \\
\hline$\%(+/-)$ & $(-80.314)$ & $(-89.44)$ & $(+25)$ \\
\hline $\mathrm{LSD}_{0.05}$ & 0.225 & 1.38 & 0.171 \\
\hline
\end{tabular}

Table 4....... ( Contd)

Set-II= 0.25ppm Ascorbic acid applied through roots in soil

\begin{tabular}{|c|c|c|c|}
\hline Treatment & $\begin{array}{c}\text { Total Root Dry Wt } \\
(\mathbf{g m s})\end{array}$ & Total Plant Dry Wt (gms) & Root Weight Ratio \\
\hline Control, Mean, SE & $0.201 \mathrm{a} \pm 0.109$ & $1.276 \mathrm{a} \pm 0.327$ & $0.248 \mathrm{a} \pm 0.178$ \\
\hline $\begin{array}{c}60 \mathrm{mM} \mathrm{NaCl}, \\
\text { Mean, SE }\end{array}$ & $0.226 \mathrm{a} \pm 0.006$ & $1.137 \mathrm{a} \pm 0.231$ & $0.214 \mathrm{a} \pm 0.04$ \\
\hline$\%(+/-)$ & $(+12.437)$ & $(-10.893)$ & $(-13.709)$ \\
\hline $\begin{array}{c}100 \mathrm{mM} \mathrm{NaCl}, \\
\text { Mean, SE }\end{array}$ & $0.0127 \mathrm{a} \pm 0.0002$ & $0.298 \mathrm{a} \pm 0.245$ & $0.213 \mathrm{a} \pm 0.132$ \\
\hline$\%(+/-)$ & $(-93.681)$ & $(-76.645)$ & $(-14.112)$ \\
\hline $\mathrm{LSD}_{0.05}$ & 0.219 & 0.964 & 0.451 \\
\hline
\end{tabular}

Table 4........ ( Contd)

Set-III= 0.50ppm Ascorbic acid applied through roots in soil

\begin{tabular}{|c|c|c|c|}
\hline Treatment & $\begin{array}{c}\text { Total Root Dry Wt } \\
(\mathbf{g m s})\end{array}$ & Total Plant Dry Wt (gms) & Root Weight Ratio \\
\hline Control, Mean, SE & $0.135 \mathrm{a} \pm 0.064$ & $1.376 \mathrm{a} \pm 0.472$ & $0.145 \mathrm{a} \pm 0.07$ \\
\hline $\begin{array}{c}60 \mathrm{mM} \mathrm{NaCl}, \\
\text { Mean, SE }\end{array}$ & $0.255 \mathrm{a} \pm 0.077$ & $1.398 \mathrm{a} \pm 0.062$ & $0.181 \mathrm{a} \pm 0.053$ \\
\hline$\%(+/-)$ & $(+88.888)$ & $(+1.598)$ & $(+24.827)$ \\
\hline $\begin{array}{c}100 \mathrm{mM} \mathrm{NaCl}, \\
\mathrm{Mean}, \mathrm{SE}\end{array}$ & $0.158 \mathrm{a} \pm 0.073$ & $2.05 \mathrm{a} \pm 0.597$ & $0.063 \mathrm{a} \pm 0.024$ \\
\hline$\%(+/-)$ & $(+17.037)$ & $(+48.982)$ & $(-56.551)$ \\
\hline $\mathrm{LSD}_{0.05}$ & 0.248 & 1.527 & 0.183 \\
\hline
\end{tabular}


Table 4........ ( Contd)

Set-IV=0.25ppm Ascorbic acid applied as a foliar spray

\begin{tabular}{|c|c|c|c|}
\hline Treatment & $\begin{array}{c}\text { Total Root Dry Wt } \\
(\mathbf{g m s})\end{array}$ & Total Plant Dry Wt (gms) & Root Weight Ratio \\
\hline Control, Mean, SE & $0.197 \mathrm{a} \pm 0.049$ & $3.278 \mathrm{a} \pm 0.759$ & $0.074 \mathrm{a} \pm 0.034$ \\
\hline $\begin{array}{c}\text { 60mM NaCl, } \\
\text { Mean, SE }\end{array}$ & $0.037 \mathrm{a} \pm 0.036$ & $0.915 \mathrm{~b} \pm 0.262$ & $0.031 \mathrm{a} \pm 0.029$ \\
\hline$\%(+/-)$ & $(-81.218)$ & $(-72.086)$ & $(-58.108)$ \\
\hline $\begin{array}{c}100 \mathrm{mM} \mathrm{NaCl}, \\
\text { Mean, SE }\end{array}$ & $0.307 \mathrm{a} \pm 0.163$ & $0.158 \mathrm{~b} \pm 0.106$ & $5.263 \mathrm{a} \pm 4.115$ \\
\hline$\%(+/-)$ & $(+55.837)$ & $(-95.179)$ & $(+70.162)$ \\
\hline LSD & 0.350 & 1.619 & 0.451 \\
\hline
\end{tabular}

Table 4........ ( Contd)

Set-V=0.50ppm Ascorbic acid applied as a foliar spray

\begin{tabular}{|c|c|c|c|}
\hline Treatment & $\begin{array}{c}\text { Total Root Dry Wt } \\
(\text { gms })\end{array}$ & Total Plant Dry Wt (gms) & Root Weight Ratio \\
\hline Control, Mean, SE & $0.307 \mathrm{a} \pm 0.012$ & $2.029 \mathrm{a} \pm 0.029$ & $0.151 \mathrm{a} \pm 0.046$ \\
\hline $\begin{array}{c}\text { 60mM NaCl, } \\
\text { Mean, SE }\end{array}$ & $0.053 \mathrm{a} \pm 0$ & $0.395 \mathrm{~b} \pm 0.007$ & $0.141 \mathrm{a} \pm 0$ \\
\hline$\%(+/-)$ & $(-82.736)$ & $(-80.532)$ & $(-6.622)$ \\
\hline $\begin{array}{c}100 \mathrm{mM} \mathrm{NaCl}, \\
\text { Mean, SE }\end{array}$ & $0.011 \mathrm{a}$ & $0.276 \mathrm{~b}$ & $0.041 \mathrm{a}$ \\
\hline$\%(+/-)$ & $(-96.416)$ & $(-86.397)$ & $(-72.847)$ \\
\hline $\mathrm{LSD}_{0.05}$ & 0.328 & 1.493 & 0.328 \\
\hline
\end{tabular}

Means followed by different letters in the same column differ significantly at $95 \%$ probability level according to New Duncan's Multiple Range Test. Figures in parentheses indicate \% promotion $(+)$ and reduction (-) over control

\section{Stem weight ratio}

Plants of set-I and set-II results increase in saline media as juxtaposition with control. Set-III and set-V resulted decreased in saline media as juxtaposition with control. Set-IV resulted decrease in $60 \mathrm{mM}$ salt stress as juxtaposition with control. Juxtaposition of set-II and set-III, set-II results a slight increase in saline media as juxtaposition with set-III. Juxtaposition of set-II and set-III with set-I, Set-II resulted

\section{Table 5. Effect of Ascorbic acid and differe} of Solanum melongena.

Set-I= Without Ascorbic acid

\begin{tabular}{|c|c|c|c|}
\hline Treatment & $\begin{array}{c}\text { Total stem dry Wt } \\
(\mathbf{g m s})\end{array}$ & Total plant dry Wt $(\mathbf{g m s})$ & Stem Weight Ratio \\
\hline Control, Mean, SE & $1.073 \mathrm{a} \pm 0.131$ & $3.021 \mathrm{a} \pm 0.42$ & $0.358 \mathrm{a} \pm 0.024$ \\
\hline $\begin{array}{c}60 \mathrm{mM} \mathrm{NaCl}, \\
\text { Mean, SE }\end{array}$ & $1.044 \mathrm{a} \pm 0.258$ & $2.142 \mathrm{a} \pm 0.518$ & $0.486 \mathrm{a} \pm 0.008$ \\
\hline$\%(+/-)$ & $(-2.702)$ & $(-29.096)$ & $(-35.754)$ \\
\hline $\begin{array}{c}100 \mathrm{mM} \mathrm{NaCl}, \\
\mathrm{Mean}, \mathrm{SE}\end{array}$ & $0.232 \mathrm{~b} \pm 0.11$ & $0.319 \mathrm{~b} \pm 0.173$ & $0.781 \mathrm{c} \pm 0.051$ \\
\hline$\%(+/-)$ & $(-78.378)$ & $(-89.44)$ & $(-118.156)$ \\
\hline $\mathrm{LSD}_{0.05}$ & 0.2 & 1.38 & 0.115 \\
\hline
\end{tabular}

increase in $60 \mathrm{mM}$ salt stress and set-III in control as juxtaposition with set-I. Juxtaposition between set-IV and set-V resulted that Set-IV showed a slight reduction in $60 \mathrm{mM}$ salt stress as juxtaposition with set-V. Juxtaposition of set-IV and set-V with set-I, both of these sets resulted increase in $60 \mathrm{mM}$ salt stress and control as juxtaposition with set-I (Table 5). 
Table 5........ ( Contd)

Set-II= 0.25ppm Ascorbic acid applied through roots in soil

\begin{tabular}{|c|c|c|c|}
\hline Treatment & $\begin{array}{c}\text { Total stem dryWt } \\
(\mathbf{g m s})\end{array}$ & Total plant dry Wt (gms) & Stem Weight Ratio \\
\hline Control, Mean, SE & $0.259 \mathrm{a} \pm 0.156$ & $1.276 \mathrm{a} \pm 0.327$ & $0.178 \mathrm{a} \pm 0.11$ \\
\hline $\begin{array}{c}\text { 60mM NaCl, } \\
\text { Mean, SE }\end{array}$ & $0.656 \mathrm{a} \pm 0.227$ & $1.137 \mathrm{a} \pm 0.231$ & $0.55 \mathrm{ab} \pm 0.078$ \\
\hline$\%(+/-)$ & $(-153.281)$ & $(-10.893)$ & $(-208.988)$ \\
\hline $\begin{array}{c}100 \mathrm{mM} \mathrm{NaCl}, \\
\text { Mean, SE }\end{array}$ & $0.279 \mathrm{a} \pm 0.242$ & $0.298 \mathrm{a} \pm 0.245$ & $0.732 \mathrm{~b} \pm 0.159$ \\
\hline$\%(+/-)$ & $(-7.722)$ & $(-76.645)$ & $(-311.235)$ \\
\hline $\mathrm{LSD}_{0.05}$ & 0.734 & 0.964 & 0.418 \\
\hline
\end{tabular}

Table 5........ ( Contd)

Set-III= 0.50ppm Ascorbic acid applied through roots in soil

\begin{tabular}{|c|c|c|c|}
\hline Treatment & $\begin{array}{c}\text { Total stem dryWt } \\
(\mathbf{g m s})\end{array}$ & Total plant dry Wt (gms) & Stem Weight Ratio \\
\hline Control, Mean, SE & $0.516 \mathrm{a} \pm 0.148$ & $1.376 \mathrm{a} \pm 0.472$ & $0.409 \mathrm{a} \pm 0.118$ \\
\hline $\begin{array}{c}\text { 60mM NaCl, } \\
\text { Mean, SE }\end{array}$ & $0.537 \mathrm{a} \pm 0.052$ & $1.398 \mathrm{a} \pm 0.062$ & $0.386 \mathrm{a} \pm 0.044$ \\
\hline$\%(+/-)$ & $(+4.069)$ & $(+1.598)$ & $(-5.623)$ \\
\hline $\begin{array}{c}100 \mathrm{mM} \mathrm{NaCl}, \\
\text { Mean, SE }\end{array}$ & $0.841 \mathrm{a} \pm 0.314$ & $2.05 \mathrm{a} \pm 0.597$ & $0.374 \mathrm{a} \pm 0.0626$ \\
\hline$\%(+/-)$ & $(+62.984)$ & $(+48.982)$ & $(-8.557)$ \\
\hline $\mathrm{LSD}_{0.05}$ & 0.702 & 1.527 & 0.282 \\
\hline
\end{tabular}

Table 5........ ( Contd)

Set-IV=0.25ppm Ascorbic acid applied as a foliar spray

\begin{tabular}{|c|c|c|c|}
\hline Treatment & $\begin{array}{c}\text { Total stem dryWt } \\
(\mathbf{g m s})\end{array}$ & Total plant dry Wt (gms) & Stem Weight Ratio \\
\hline Control, Mean, SE & $1.6 \pm 0.452$ & $3.278 \mathrm{a} \pm 0.759$ & $0.484 \mathrm{a} \pm 0.047$ \\
\hline $\begin{array}{c}\text { 60mM NaCl, } \\
\text { Mean, SE }\end{array}$ & $0.465 \mathrm{~b} \pm 0.155$ & $0.915 \mathrm{~b} \pm 0.262$ & $0.546 \mathrm{ab} \pm 0.122$ \\
\hline$\%(+/-)$ & $(-70.9375)$ & $(-72.086)$ & $(+12.809)$ \\
\hline $\begin{array}{c}100 \mathrm{mM} \mathrm{NaCl}, \\
\text { Mean, SE }\end{array}$ & $0.049 \mathrm{~b} \pm 0.038$ & $0.158 \mathrm{~b} \pm 0.106$ & $0.258 \mathrm{~b} \pm 0.04$ \\
\hline$\%(+/-)$ & $(-96.9375)$ & $(-95.179)$ & $(-46.694)$ \\
\hline $\mathrm{LSD}_{0.05}$ & 0.958 & 1.619 & 0.274 \\
\hline
\end{tabular}

Table 5........ ( Contd)

Set-V=0.50 ppm Ascorbic acid applied as a foliar spray

\begin{tabular}{|c|c|c|c|}
\hline Treatment & $\begin{array}{c}\text { Total stem dry Wt } \\
(\text { gms })\end{array}$ & Total plant dry Wt (gms) & Stem Weight Ratio \\
\hline Control, Mean, SE & $1.391 \mathrm{a} \pm 0.67$ & $2.029 \mathrm{a} \pm 0.747$ & $0.601 \mathrm{a} \pm 0.147$ \\
\hline $\begin{array}{c}\text { 60mM NaCl, } \\
\text { Mean, SE }\end{array}$ & $0.197 \mathrm{a} \pm 0.021$ & $0.395 \mathrm{~b} \pm 0.029$ & $0.514 \mathrm{a} \pm 0.101$ \\
\hline$\%(+/-)$ & $(-85.837)$ & $(-80.532)$ & $(-14.475)$ \\
\hline $\begin{array}{c}100 \mathrm{mM} \mathrm{NaCl}, \\
\text { Mean, SE }\end{array}$ & $0.131 \mathrm{a} \pm 0.003$ & $0.276 \mathrm{~b} \pm 0.007$ & $0.476 \mathrm{a} \pm 0$ \\
\hline$\%(+/-)$ & $(-90.582)$ & $(-86.397)$ & $(-20.798)$ \\
\hline $\mathrm{LSD}_{0.05}$ & 1.341 & 1.493 & 0.357 \\
\hline
\end{tabular}

Means followed by different letters in the same column differ significantly at $95 \%$ probability level according to New Duncan's Multiple Range Test. Figures in parentheses indicate \% promotion (+) and reduction (-) over control 


\section{Leaf weight ratio}

Plants of set-I and set-II results decrease in saline media as juxtaposition with control. Set-III and set-IV exhibited increase in $100 \mathrm{mM}$ salt stress as juxtaposition with control. Set-V resulted increase in saline media as juxtaposition with control. Juxtaposition of set-II and set-III, set-II resulted increase in control as juxtaposition with set-III. Comparison of set-II and set-
III with set-I, Set-II resulted increase in control and set-III exhibited increase in saline media as juxtaposition with set-I. Juxtaposition between set-IV and set-V resulted that Set-V showed increase in $60 \mathrm{mM}$ salt stress as juxtaposition with setIV. Juxtaposition of set-IV and set-V with set-I, both of these sets resulted increase in $100 \mathrm{mM}$ salt stress and control juxtaposition with set-I (Table 6).

Table 6. Effects of Ascorbic acid and different $\mathrm{NaCl}$ concentrations on leaf weight ratio of Solanum melongena.

Set-I= Without Ascorbic acid

\begin{tabular}{|c|c|c|c|}
\hline Treatment & $\begin{array}{c}\text { Total leaves dry Wt } \\
(\mathbf{g m s})\end{array}$ & Total plant dry Wt (gms) & Leaf Weight Ratio \\
\hline Control, Mean, SE & $1.566 \mathrm{a} \pm 0.268$ & $3.021 \mathrm{a} \pm 0.42$ & $0.517 \mathrm{a} \pm 0.035$ \\
\hline $\begin{array}{c}60 \mathrm{mM} \mathrm{NaCl}, \\
\text { Mean, SE }\end{array}$ & $0.901 \mathrm{a} \pm 0.316$ & $2.142 \mathrm{a} \pm 0.518$ & $0.4 \mathrm{a} \pm 0.042$ \\
\hline$\%(+/-)$ & -42.464 & -29.096 & -22.630 \\
\hline $\begin{array}{c}100 \mathrm{mM} \mathrm{NaCl}, \\
\text { Mean, SE }\end{array}$ & $0.012 \mathrm{~b} \pm 0$ & $0.319 \mathrm{~b} \pm 0.173$ & $0.062 \mathrm{~b} \pm 0.021$ \\
\hline$\%(+/-)$ & -99.233 & -89.440 & -88.007 \\
\hline $\mathrm{LSD}_{0.05}$ & 0.829 & 1.38 & 0.119 \\
\hline
\end{tabular}

Table 6........ ( Contd)

Set-II= 0.25ppm Ascorbic acid applied through roots in soil

\begin{tabular}{|c|c|c|c|}
\hline Treatment & $\begin{array}{c}\text { Total leaves dry Wt } \\
(\mathbf{g m s})\end{array}$ & Total plant dry Wt (gms) & Leaf Weight Ratio \\
\hline Control, Mean, SE & $0.8147 \mathrm{a} \pm 0.38$ & $1.276 \mathrm{a} \pm 0.327$ & $1.277 \mathrm{a} \pm 0.150$ \\
\hline $\begin{array}{c}\text { 60mM NaCl, } \\
\text { Mean, SE }\end{array}$ & $0.253 \mathrm{ab} \pm 0.054$ & $1.137 \mathrm{a} \pm 0.231$ & $0.235 \mathrm{~b} \pm 0.060$ \\
\hline$\%(+/)$ & -68.945 & -10.893 & -81.597 \\
\hline $\begin{array}{c}100 \mathrm{mM} \mathrm{NaCl}, \\
\text { Mean, SE }\end{array}$ & $0.006 \mathrm{~b} \pm 0.003$ & $0.298 \mathrm{a} \pm 0.245$ & $0.053 \mathrm{~b} \pm 0.027$ \\
\hline$\%(+/-)$ & -99.263 & -76.645 & -95.849 \\
\hline $\mathrm{LSD}_{0.05}$ & 0.769 & 0.964 & 0.329 \\
\hline
\end{tabular}

Table 6........ ( Contd)

Set-III= 0.50ppm Ascorbic acid applied through roots in soil

\begin{tabular}{|c|c|c|c|}
\hline Treatment & $\begin{array}{c}\text { Total leaves dry Wt } \\
(\mathbf{g m s})\end{array}$ & Total plant dry Wt (gms) & Leaf Weight Ratio \\
\hline Control, Mean, SE & $0.725 \mathrm{a} \pm 0.42$ & $1.376 \mathrm{a} \pm 0.472$ & $0.445 \mathrm{a} \pm 0.148$ \\
\hline $\begin{array}{c}\text { 60mM NaCl, } \\
\text { Mean, SE }\end{array}$ & $0.604 \mathrm{a} \pm 0.034$ & $1.398 \mathrm{a} \pm 0.062$ & $0.432 \mathrm{a} \pm 0.011$ \\
\hline$\%(+/)$ & $(-16.689)$ & $(+1.598)$ & $(-2.921)$ \\
\hline $\begin{array}{c}100 \mathrm{mM} \mathrm{NaCl}, \\
\text { Mean, SE }\end{array}$ & $1.05 \mathrm{a} \pm 0.209$ & $2.05 \mathrm{a} \pm 0.597$ & $0.562 \mathrm{a} \pm 0.087$ \\
\hline$\%(+/-)$ & $(+44.827)$ & $(-48.982)$ & $(+26.292)$ \\
\hline $\mathrm{LSD}_{0.05}$ & 0.941 & 1.527 & 0.297 \\
\hline
\end{tabular}


Table 6........ ( Contd)

Set-IV=0.25 ppm Ascorbic acid applied as a foliar spray

\begin{tabular}{|c|c|c|c|}
\hline Treatment & $\begin{array}{c}\text { Total leaves dry Wt } \\
(\text { gms })\end{array}$ & Total plant dry Wt (gms) & Leaf Weight Ratio \\
\hline Control, Mean, SE & $0.831 \mathrm{a} \pm 0.217$ & $3.278 \mathrm{a} \pm 0.759$ & $0.266 \mathrm{a} \pm 0.051$ \\
\hline $\begin{array}{c}\text { 60mM NaCl, } \\
\text { Mean, SE }\end{array}$ & $0.252 \mathrm{~b} \pm 0.149$ & $0.915 \mathrm{~b} \pm 0.262$ & $0.229 \mathrm{ab} \pm 0.128$ \\
\hline$\%(+/-)$ & $(-69.675)$ & $(-72.086)$ & $(-13.909)$ \\
\hline $\begin{array}{c}100 \mathrm{mM} \mathrm{NaCl}, \\
\text { Mean, SE }\end{array}$ & $0.071 \mathrm{~b} \pm 0.032$ & $0.158 \mathrm{~b} \pm 0.106$ & $0.625 \mathrm{~b} \pm 0.131$ \\
\hline$\%(+/-)$ & $(-91.456)$ & $(-95.179)$ & $(+134.962)$ \\
\hline $\mathrm{LSD}_{0.05}$ & 0.533 & 1.619 & 0.381 \\
\hline
\end{tabular}

Table 6........ ( Contd)

Set-V = 0.50 ppm Ascorbic acid applied as a foliar spray

\begin{tabular}{|c|c|c|c|}
\hline Treatment & $\begin{array}{c}\text { Total leaves dry Wt } \\
(\mathbf{g m s})\end{array}$ & Total plant dry Wt (gms) & Leaf Weight Ratio \\
\hline Control, Mean, SE & $0.33 \mathrm{a} \pm 0.062$ & $2.029 \mathrm{a} \pm 0.747$ & $0.247 \mathrm{a} \pm 0.123$ \\
\hline $\begin{array}{c}60 \mathrm{mM} \mathrm{NaCl}, \\
\text { Mean, SE }\end{array}$ & $0.144 \mathrm{~b} \pm 0.064$ & $0.395 \mathrm{~b} \pm 0.029$ & $0.343 \mathrm{a} \pm 0.147$ \\
\hline$\%(+/-)$ & $(-56.363)$ & $(-80.532)$ & $(+38.866)$ \\
\hline $\begin{array}{c}100 \mathrm{mM} \mathrm{NaCl}, \\
\text { Mean, SE }\end{array}$ & $0.133 \mathrm{~b} \pm 0.003$ & $0.276 \mathrm{~b} \pm 0.007$ & $0.481 \pm 0$ \\
\hline$\%(+/-)$ & -59.696 & -86.397 & -94.736 \\
\hline $\mathrm{LSD}_{0.05}$ & 1.799 & 1.493 & 0.385 \\
\hline
\end{tabular}

Means followed by different letters in the same column differ significantly at $95 \%$ probability level according to New Duncan's Multiple Range Test. Figures in parentheses indicate \% promotion (+) and reduction (-) over control

\section{Specific leaf area ratio}

Plants of set-I and set-II results increase in $100 \mathrm{mM}$ salt stress as juxtaposition with control. Set-III resulted increase in control as juxtaposition with saline media. Set-IV resulted prominent reduction in control as juxtaposition with saline media. Set-V resulted increase in $60 \mathrm{mM}$ salt stress as juxtaposition with control. Juxtaposition of set-II and set-III, set-II resulted increase in saline media as juxtaposition with set-III.
Comparison of set-II and set-III with set-I, Set-II resulted increase in both salinity treatments and control while set-III exhibited decrease in $100 \mathrm{mM}$ salt stress as juxtaposition with set-I. Juxtaposition between set-IV and set-V resulted that SetIV resulted increase in $100 \mathrm{mM}$ salt stress as juxtaposition with set-V. Juxtaposition of set-IV and set-V with set-I, both sets resulted increase in control and $60 \mathrm{mM}$ salt stress juxtaposition with set-I (Table 7).

Table 7. Effect of Ascorbic acid and different $\mathrm{NaCl}$ concentrations on leaf area ratio of Solanum melongena.

Set-I= Without Ascorbic acid

\begin{tabular}{|c|c|c|c|}
\hline Treatment & Leaf Area $(\mathbf{m m 2})$ & Total plant dry Wt (gms) & Leaf Area Ratio \\
\hline Control, Mean, SE & $76.84 \mathrm{a} \pm 40.535$ & $1.566 \mathrm{a} \pm 0.268$ & $46.216 \mathrm{a} \pm 22.694$ \\
\hline $\begin{array}{c}60 \mathrm{mM} \mathrm{NaCl}, \\
\text { Mean, SE }\end{array}$ & $76.442 \mathrm{a} \pm 14.062$ & $0.901 \mathrm{a} \pm 0.316$ & $134.204 \mathrm{~b} \pm 29.299$ \\
\hline$\%(+/-)$ & $(-0.517)$ & $(-42.464)$ & $(-190.384)$ \\
\hline $\begin{array}{c}100 \mathrm{mM} \mathrm{NaCl}, \\
\mathrm{Mean}, \mathrm{SE}\end{array}$ & $23.881 \mathrm{a} \pm 11.134$ & $0.012 \mathrm{~b} \pm 0$ & $276.561 \mathrm{~b} \pm 819.04$ \\
\hline$\%(+/-)$ & $(-68.921)$ & $(-99.233)$ & $(-589.481)$ \\
\hline $\mathrm{LSD}_{0.05}$ & 88.56 & 0.829 & 163.03 \\
\hline
\end{tabular}


Table 7...........(contd)

Set-II= 0.25 ppm Ascorbic acid applied through roots in soil

\begin{tabular}{|c|c|c|c|}
\hline Treatment & Leaf Area $(\mathbf{m m} 2)$ & Total plant dry Wt (gms) & Leaf Area Ratio \\
\hline Control, Mean, SE & $85.669 \mathrm{a} \pm 12.234$ & $0.8147 \mathrm{a} \pm 0.38$ & $173.546 \mathrm{a} \pm 91.862$ \\
\hline $\begin{array}{c}60 \mathrm{mM} \mathrm{NaCl}, \\
\text { Mean, SE }\end{array}$ & $149.675 \mathrm{ab} \pm 37.748$ & $0.253 \mathrm{ab} \pm 0.054$ & $700.95 \mathrm{~b} \pm 261.396$ \\
\hline$\%(+/-)$ & $(+74.713)$ & $(-68.945)$ & $(+303.898)$ \\
\hline $\begin{array}{c}100 \mathrm{mM} \mathrm{NaCl}, \\
\mathrm{Mean}, \mathrm{SE}\end{array}$ & $38.44 \mathrm{~b} \pm 17.229$ & $0.006 \mathrm{~b} \pm 0.003$ & $6586.717 \mathrm{~b} \pm 572.763$ \\
\hline$\%(+/-)$ & $(-55.129)$ & $(-99.263)$ & $(+369.372)$ \\
\hline $\mathrm{LSD}_{0.05}$ & 86.43 & 0.769 & 127.18 \\
\hline
\end{tabular}

Table $7 \ldots . . . . . .($ contd)

Set-III= 0.50 ppm Ascorbic acid applied through roots in soil

\begin{tabular}{|c|c|c|c|}
\hline Treatment & Leaf Area $(\mathbf{m m} 2)$ & Total plant dry Wt $(\mathbf{g m s})$ & Leaf Area Ratio \\
\hline Control, Mean, SE & $68.981 \mathrm{a} \pm 22.263$ & $0.725 \mathrm{a} \pm 0.42$ & $187.787 \mathrm{a} \pm 112.687$ \\
\hline $\begin{array}{c}60 \mathrm{mM} \mathrm{NaCl}, \\
\text { Mean, SE }\end{array}$ & $82.238 \mathrm{a} \pm 10.212$ & $0.604 \mathrm{a} \pm 0.034$ & $137.260 \mathrm{a} \pm 20.543$ \\
\hline$\%(+/-)$ & $(+19.218)$ & $(-16.689)$ & $(-26.906)$ \\
\hline $\begin{array}{c}100 \mathrm{mM} \mathrm{NaCl}, \\
\text { Mean, SE }\end{array}$ & $60.344 \mathrm{a} \pm 30.22$ & $1.05 \mathrm{a} \pm 0.21$ & $47.98 \mathrm{a} \pm 23.965$ \\
\hline$\%(+/-)$ & $(-12.52)$ & $(+44.827)$ & $(-74.45)$ \\
\hline $\mathrm{LSD}_{0.05}$ & 77.717 & 0.941 & 233.805 \\
\hline
\end{tabular}

Table 7..........(contd)

Set-IV=0.25 ppm Ascorbic acid applied as a foliar spray

\begin{tabular}{|c|c|c|c|}
\hline Treatment & Leaf Area $(\mathbf{m m 2})$ & Total plant dry Wt (gms) & Leaf Area Ratio \\
\hline $\begin{array}{c}\text { Control, Mean, SE } \\
122.999 \mathrm{a} \pm 37.023\end{array}$ & $0.831 \mathrm{a} \pm 0.217$ & $144.693 \mathrm{a} \pm 9.406$ \\
\hline $\begin{array}{c}\text { 60mM NaCl, } \\
\text { Mean, SE }\end{array}$ & $57.197 \mathrm{a} \pm 28.65$ & $0.252 \mathrm{~b} \pm 0.149$ & $1487.466 \mathrm{a} \pm 139.11$ \\
\hline$\%(+/-)$ & $(-53.497)$ & $(-69.675)$ & $(+928.015)$ \\
\hline $\begin{array}{c}100 \mathrm{mM} \mathrm{NaCl}, \\
\mathrm{Mean}, \mathrm{SE}\end{array}$ & $48.298 \mathrm{a} \pm 5.193$ & $0.071 \mathrm{~b} \pm 0.032$ & $1016.960 \mathrm{a} \pm 371.27$ \\
\hline$\%(+/-)$ & $(-60.733)$ & $(-91.456)$ & $(+602.839)$ \\
\hline $\mathrm{LSD}_{0.05}$ & 94.098 & 0.533 & 2878.557 \\
\hline
\end{tabular}

Table 7.........(contd)

Set-V=0.50 ppm Ascorbic acid applied as a foliar spray

\begin{tabular}{|c|c|c|c|}
\hline Treatment & Leaf Area $(\mathbf{m m})$ & Total plant dry Wt $(\mathbf{g m s})$ & Leaf Area Ratio \\
\hline Control, Mean, SE & $76.441 \mathrm{a} \pm 15.902$ & $0.33 \mathrm{a} \pm 0.062$ & $228.998 \mathrm{a} \pm 13.44$ \\
\hline $\begin{array}{c}60 \mathrm{mM} \mathrm{NaCl}, \\
\mathrm{Mean}, \mathrm{SE}\end{array}$ & $79.088 \mathrm{a} \pm 2.349$ & $0.144 \mathrm{~b} \pm 0.064$ & $1792.209 \mathrm{a} \pm 1402.785$ \\
\hline$\%(+/-)$ & $(+3.462)$ & $(-56.363)$ & $(+682.63)$ \\
\hline $\begin{array}{c}100 \mathrm{mM} \mathrm{NaCl}, \\
\mathrm{Mean}, \mathrm{SE}\end{array}$ & $50.717 \mathrm{a} \pm 5.772$ & $0.133 \mathrm{~b} \pm 0.003$ & $378.318 \mathrm{a} \pm 34.351$ \\
\hline$\%(+/-)$ & $(-33.652)$ & $(-59.696)$ & $(-65.205)$ \\
\hline $\mathrm{LSD}_{0.05}$ & 34.124 & 1.799 & 280.474 \\
\hline
\end{tabular}

Means followed by different letters in the same column differ significantly at $95 \%$ probability level according to New Duncan's Multiple Range Test. Figures in parentheses indicate \% promotion $(+)$ and reduction (-) over control

\section{Leaf area ratio}

Plants of set-I and set-V resulted prominent increase in $100 \mathrm{mM}$ salt stress as juxtaposition with control. Set-II resulted decrease in control as juxtaposition with salt stress. Set-III resulted prominent increase in control as juxtaposition with salt stress. Set-IV resulted prominent increase 
in $60 \mathrm{mM}$ salt stress as juxtaposition with control. Juxtaposition of set-II and set-III, set-II resulted increase in control as well as in saline media as juxtaposition with set-III. Juxtaposition of set-II and set-III with set-I, Set-II resulted reduction in $60 \mathrm{mM}$ salt stress, while set-III resulted increase in control as juxtaposition with set-I. Juxtaposition between set-IV and set-V resulted that Set-IV resulted increase in control and in $60 \mathrm{mM}$ salt stress as juxtaposition with set-V. Juxtaposition of set-IV and set-V with set-I, Set-IV resulted increase in control and in $60 \mathrm{mM}$ salt stress and set- $\mathrm{V}$ showed increase both in control and salinity treated plants as juxtaposition with set-I (Table 8).

Table 8. Effect of Ascorbic acid and different $\mathrm{NaCl}$ concentrations on specific leaf area of Solanum melongena.

Set-I= Without Ascorbic acid

\begin{tabular}{|c|c|c|c|}
\hline Treatment & Leaf Area $(\mathbf{m m})$ & Total leaf dry Wt $(\mathbf{g m s})$ & Specific Leaf Area \\
\hline Control, Mean, SE & $76.84 \mathrm{a} \pm 40.535$ & $3.021 \mathrm{a} \pm 0.42$ & $231.947 \mathrm{a} \pm 116.921$ \\
\hline $\begin{array}{c}\text { 60mM NaCl, Mean, } \\
\text { SE }\end{array}$ & $76.442 \mathrm{a} \pm 14.062$ & $2.142 \mathrm{a} \pm 0.518$ & $728.255 \mathrm{a} \pm 351.33$ \\
\hline$\%(+/-)$ & -0.518 & -29.096 & 213.975 \\
\hline $\begin{array}{c}100 \mathrm{mM} \mathrm{NaCl,} \mathrm{Mean,} \\
\mathrm{SE}\end{array}$ & $23.881 \mathrm{a} \pm 11.134$ & $0.319 \mathrm{~b} \pm 0.173$ & $1416.216 \mathrm{a} \pm 565.7$ \\
\hline$\%(+/-)$ & -68.921 & -89.441 & 510.577 \\
\hline $\mathrm{LSD}_{0.05}$ & 88.56 & 1.38 & 135.79 \\
\hline
\end{tabular}

Table $8 \ldots \ldots \ldots . . .($ contd $)$

Set-II= 0.25 ppm Ascorbic acid applied through roots in soil

\begin{tabular}{|c|c|c|c|}
\hline Treatment & Leaf Area $(\mathbf{m m})$ & Total leaf dry Wt $($ gms $)$ & Specific Leaf Area \\
\hline Control, Mean, SE & $85.669 \mathrm{a} \pm 12.234$ & $1.276 \mathrm{a} \pm 0.327$ & $3064.291 \mathrm{a} \pm 279.801$ \\
\hline $60 \mathrm{mM} \mathrm{NaCl}$, Mean, SE & $149.675 \mathrm{ab} \pm 37.748$ & $1.137 \mathrm{a} \pm 0.231$ & $652.927 \mathrm{~b} \pm 152.998$ \\
\hline$\%(+/-)$ & 74.713 & -10.893 & -78.692 \\
\hline $\begin{array}{c}100 \mathrm{mM} \mathrm{NaCl}, \text { Mean, } \\
\text { SE }\end{array}$ & $38.44 \mathrm{~b} \pm 17.229$ & $0.298 \mathrm{a} \pm 0.245$ & $3047.449 \mathrm{~b} \pm 139.215$ \\
\hline$\%(+/-)$ & -55.130 & -76.646 & -0.550 \\
\hline LSD $_{0.05}$ & 86.43 & 0.964 & 127.18 \\
\hline
\end{tabular}

Table 8...........(contd)

Set-III=0.50 ppm Ascorbic acid applied through roots in soil

\begin{tabular}{|c|c|c|c|}
\hline Treatment & Leaf Area $(\mathbf{m m})$ & Total leaf dry Wt $(\mathbf{g m s})$ & Specific Leaf Area \\
\hline Control, Mean, SE & $68.981 \mathrm{a} \pm 22.263$ & $1.376 \mathrm{a} \pm 0.472$ & $3031.187 \mathrm{a} \pm 277.658$ \\
\hline $\begin{array}{c}60 \mathrm{mM} N a C l, M e a n \\
\text { SE }\end{array}$ & $82.238 \mathrm{a} \pm 10.212$ & $1.398 \mathrm{a} \pm 0.062$ & $389.377 \mathrm{a} \pm 112.151$ \\
\hline$\%(+/-)$ & $(+19.218)$ & $(+1.598)$ & $(-87.154)$ \\
\hline $\begin{array}{c}100 \mathrm{mM} ~ \\
\text { SECl, Mean, }\end{array}$ & $60.344 \mathrm{a} \pm 30.219$ & $2.05 \mathrm{a} \pm 0.597$ & $265.765 \mathrm{a} \pm 124.87$ \\
\hline$\%(+/-)$ & $(-12.520)$ & $(+48.982)$ & $(-91.232)$ \\
\hline $\mathrm{LSD}_{0.05}$ & 77.717 & 1.527 & 233.805 \\
\hline
\end{tabular}

Table $8 \ldots . . . . . . .($ contd)

Set-IV=0.25 ppm Ascorbic acid applied as a foliar spray

\begin{tabular}{|c|c|c|c|}
\hline Treatment & Leaf Area $(\mathrm{mm} 2)$ & Total leaf dry Wt $(\mathrm{gms})$ & Specific Leaf Area \\
\hline Control, Mean, SE & $122.999 a \pm 37.023$ & $3.278 \mathrm{a} \pm 0.759$ & $889.056 \mathrm{a} \pm 534.48$ \\
\hline 60mM NaCl, Mean, SE & $57.197 \mathrm{a} \pm 28.644$ & $0.915 b \pm 0.262$ & $\begin{array}{c}45081.317 \mathrm{a} \\
\pm 229.78\end{array}$ \\
\hline$\%(+/-)$ & $(-53.497)$ & $(-72.086)$ & $(+4970.694)$ \\
\hline $100 \mathrm{mM} \mathrm{NaCl}$, Mean, SE & $48.298 a \pm 5.193$ & $0.158 \mathrm{~b} \pm 0.106$ & $277.699 \mathrm{a} \pm 140.81$ \\
\hline$\%(+/-)$ & $(-60.733)$ & $(-95.179)$ & $(-68.764)$ \\
\hline $\mathrm{LSD}_{0.05}$ & 94.098 & 1.619 & 287.56 \\
\hline
\end{tabular}


Table 8...........(contd)

Set-V=0.50 ppm Ascorbic acid applied as a foliar spray

\begin{tabular}{|c|c|c|c|}
\hline Treatment & Leaf Area $(\mathbf{m m})$ & Total leaf dry Wt $(\mathbf{g m s})$ & Specific Leaf Area \\
\hline Control, Mean, SE & $76.441 \mathrm{a} \pm 15.902$ & $2.029 \mathrm{a} \pm 0.747$ & $485.92 \mathrm{a} \pm 235.38$ \\
\hline $60 \mathrm{mM} \mathrm{NaCl}$, Mean, SE & $79.088 \mathrm{a} \pm 2.349$ & $0.395 \mathrm{~b} \pm 0.029$ & $1656.158 \mathrm{a} \pm 358.634$ \\
\hline$\%(+/-)$ & $(+3.462)$ & $(-80.532)$ & $(+240.829)$ \\
\hline $100 \mathrm{mM} \mathrm{NaCl}$, Mean, SE & $50.717 \mathrm{a} \pm 5.772$ & $0.276 \mathrm{~b} \pm 0.007$ & $4378.633 \mathrm{a} \pm 408.992$ \\
\hline$\%(+/-)$ & $(-33.652)$ & $(-86.397)$ & -801.101 \\
\hline $\mathrm{LSD}_{0.05}$ & 34.124 & 1.493 & 280.48 \\
\hline
\end{tabular}

Means followed by different letters in the same column differ significantly at $95 \%$ probability level according to New Duncan's Multiple Range Test. Figures in parentheses indicate \% promotion (+) and reduction (-) over control

\section{Conclusion}

Salinity has negative effects on plant growth and development which were significantly improved by exogenous and foliar spray applications of ascorbic acid. Ascorbic acid overcomes the destructive effects of salinity and its applications by either way viz. foliar spray and applied through roots in soil mostly led to increase the plant morphological, physiological and biochemical parameters and plants yield. However, the application of ascorbic acid applied in soil was more effective than application through as a foliar spray. This study justifies further work on Solanum melongena plant under a broader range of field conditions to evaluate the possibility of using ascorbic acid for improving their growth.

\section{Authors' contributions}

Conceived, designed and performed the experiments: M Irfan, Analysed the data: Nabeela, Contributed materials, analysis, tools: M Ilyas, Wrote the manuscript: KU Rahman.

\section{References}

1. Afzal I, Basra SMA, Hameed A \& Farooq M (2006). Physiological enhancements for alleviation of salt stress in wheat. Pak J Bot 38: 16491659.

2. Alam MZ, Stuchbury TR, Naylor EL \& Rashid MA (2004). Effect of salinity on growth of some modern rice cultivar. J Agron 3(1): 1-10.

3. Al-Karaki GN (2000). Growth of mycorrhizal tomato and mineral acquisition under salt stress. Mycorrhiza 10: 51-54.

4. Alscher RG, Donahue JL \& Cramer CL (1997). Reactive oxygen species and antioxitants: relationship in green cells. Physiol Plant 100: 224-233.

5. Afzal I, Basra SMA, Farooq $M$ \& Nawaz A (2006). Alleviation of salinity stress in spring wheat by hormonal priming with $\mathrm{ABA}$, salicylic acid and ascorbic acid. Inter J Agr Biol 8: 23-28.

6. Akram NA, Shafiq F \& Ashraf M (2017). Ascorbic acid a potential oxidant scavenger and its role in plant development and Abiotic stress tolerance. Front in Pl Sci 8: 613

7. Alam MZ, Stuchbury TR, Naylor EL \& Rashid MA (2004). Effect of salinity on growth of some modern rice cultivar. J Agron 3(1): 1-10.

8. Arafa AA, Khafagy MA \& El-Banna MF (2009). The effect of glycine betaine or ascorbic acid on grain germination and leaf structure of sorghum plants grown under salinity stress. Aust J Crop Sci 3:294-304.

9. Azooz MM \& Al-Fredan MA (2009). The inductive role of vitamin $\mathrm{C}$ and its mode of application on growth, water status, antioxidant enzyme activities and protein patterns of Vicia faba L. Hassawi grown under seawater irrigation. Am J Plant Physiol 4: 38-51.

10. Barh D, Srivastava HC \& Mazumdar BC (2008). Self-fruit extract and Vitamin-C improves tomato seed 
germination. J Appl Sci Res 4: 156165.

11. Burguieres E, Mccue P, Kwon Y \& Shetty K (2007). Effect of vitamin C and folic acid on seed vigor response and phenolic-linked antioxidant activity. Bioresour Technol 98: 13931404.

12. Davey MW, Mantagu MV, Dirk IMaite S, Angelos K, Smirn off N, Binenzie IJJ, Strain JJ, Favell D \& Fletcher J (2000). Plant ascorbic acid chemistry, function, metabolism, bio availability and effects of processing. J Sci Food and Agri 80:825-850.

13. Duan $\mathrm{D}, \mathrm{Li} \mathrm{W}$, Ouyang $\mathrm{H} \& \mathrm{An} \mathrm{P}$ (2007). Seed germination and seedling growth of Suaeda salsa under salt stress. Ann Bot Fennici 44: 161-169

14. Amacher J, Koenig R \& Kitchen B (2000). Salinity and plant tolerance. Electronic publishing No. AG-SO-03, Utah University Extension, Utah, USA.

15. Ashraf M (2009). Biotechnological approach of improving plant salt tolerance using antioxidants as markers. Biotech Adv 27: 84-93.

16. Baraka DM (2008). Osmotic adjustment of wheat grain germination to hyperosmotic saline by nicotine hormone. Res J Agric and Biol Sci 4: 824- 831.

17. Bartels D \& R Sunkar (2005). Drought and salt tolerance in plants. Crit. Rev. Plant Sci 24: 23-58

18. Moffat AS (2002). Finding new ways to protect drought-stricken plants. Sci Magazine.

19. Irfan M, Alam J, Ahmad I, Ali I \& Gul $H$ (2017). Effects of exogenous and foliar applications of Brassinosteroid (BRs) and salt stress on the growth, yield and physiological parameters of Lycopersicon esculentum (Mill.). Plant Sci Today 4(3): 88-101.

20. Irfan M, Alam J, Jan G \& Gul H (2018). Influence of brassinosteroid (BRs) in roots and foliar spray against salinity on physiological parameters and micro nutrients upon tomato (Lycopersicon esculentum Mill.) Science Arena Publications Spec J Bio Sci 4(2): 18-27

21. Irfan M \& Ali I (2018). The effect of heat stress on Morpho physiological traits of Triticum aestivum $\mathrm{L}$. genotypes. Science Arena Publications Spec J Agri Sci 4(1): 13-23. 